\title{
SUI FASCI DI CUBICHE A MODULO COSTANTE.
}

\author{
Memoria di Oscar Chisini (Bologna).
}

Adunanza del 13 febbraio 1916.

$$
\oint 1 \text {. }
$$

\section{Introduzione.}

Come è noto, da un punto $O$ di una cubica piana

$$
f_{3}=0
$$

escono quattro tangenti alla cubica stessa, il cui birapporto, chiamato modulo della cubica, resta costante qualunque sia la posizione assunta dal punto $O$. Se $a$ è uno dei sei valori che puó assumere questo birapporto (dipendentemente dall'ordine in cui si prendono le quattro tangenti), l'espressione

$$
J=\frac{4\left(1-\alpha+\alpha^{2}\right)^{3}}{(\alpha+\mathrm{I})^{2}(1-2 \alpha)^{2}(2-\alpha)^{2}},
$$

che è razionale nei coefficienti della quaterna di tangenti e indipendente dalla posizione del punto $O$, riesce anche razionale nei coefficienti della cubica $f_{3}$ : essa costituisce un invariante della forma $f_{3}$, anzi l'unico invariante assoluto.

Espresso per i coefficienti di $f_{3}$, l'invariante $J$ è una funzione razionale del dodicesimo grado del tipo

$$
J=\frac{S^{3}}{T^{2}}
$$

dove $S$ e $T$ sono due invarianti (relativi) rispettivamente del quarto e del sesto grado.

L'eguaglianza

$$
S=0, \quad \text { ciò } \quad J=0,
$$

esprime che la cubica $f_{3}$ è equianarmonica, cioè che tale è la quaterna di tangenti uscenti da un suo punto $O$; invece

$$
T=0, \quad \text { cioè } \quad J=\infty,
$$

esprime che la cubica è armonica, cioè che tale è la quaterna delle suddette tangenti; infine

$$
S^{3}-T^{2}=\mathrm{o}, \quad \text { ciò } \quad J=\mathrm{I}
$$

esprime che la cubica ha un punto doppio. 
Poichè - come abbiamo ricordato - $J$ contiene al dodicesimo grado i coefficienti della cubica $f_{3}=0$, cosi in ogni fascio vi sono i 2 cubiche per cui l'invariante $J$ ha un valore, generico, assegnato: ve ne sono invece solo 4 delle cquianarmoniche e 6 delle armoniche, ciascuna delle quali conta rispettivamente tre e due volte nel gruppo delle I 2 cubiche per cui l'invariante ha il valore $J=0$ e $J=\infty$.

Ora facili esempi mostrano l'esistenza di fasci di cubiche di ugual modulo, e quindi di ugual invariante, per cui l'equazione $J=K$ riesce identicamente soddisfatta : tali sono $\mathrm{i}$ fasci di cubiche con un punto base doppio, oppure $\mathrm{i}$ fasci

$$
y^{2}-\lambda\{(x-a)(x-b)(x-c)\}=0,
$$

nei quali sono fisse le tangenti che partono dal punto all'infinito dell'asse $y$. Un esempio più notevole e riposto di fasci di cubiche a modulo costante fu incontrato da S. KaNTOR nell'esame delle proiettività che lasciano fermo un fascio di cubiche ${ }^{\mathbf{x}}$ ). Egli osserva che, data una proiettività $\omega$, ciclica del terz'ordine, di punti uniti $A, B, C$, le cubiche passanti per $A, B, C$, tangenti ivi alle rette $A B, B C, C A$, e passanti ulteriormente per i tre punti $P, Q, R$ di un ciclo di $\omega$, formano un fascio di cubiche tutte invarianti per la $\omega$, le quali risultano equianarmoniche, essendo-come è noto-proprietà caratteristica di una cubica equianarmonica restare invariata per una proiettività ciclica del terz'ordine coi punti uniti sulla cubica stessa ${ }^{2}$ ).

Ma questi fasci rientrano come casi particolari in famiglie più generali di fasci di cubiche a modulo costante, la cui esistenza è messa in luce anche da un facile computo di costanti che riesce più chiaro col linguaggio della geometria astratta.

Le cubiche del piano costituiscono $\mathrm{i}$ punti di un $S_{9}$ le cui rette rappresentano i fasci di cubiche; le cubiche di dato invariante (generico) formano una varjetà $V_{8}^{12}$ del dodicesimo grado: le rette del $S_{9}$ essendo $\infty^{16}$, e occorrendo I 3 condizioni perchè una retta appartenga alla $V_{8}^{12}$, si vede che esistono almeno $\infty^{3}$ fasci di cubiche per cui l'invariante ha un valore assegnato; analogamente, poichè le cubiche equianarmoniche formano una $V_{8}^{4}$ (tripla) e le armoniche una $V_{8}^{6}$ (doppia) esisteranno (almeno) $\infty^{15}$ fasci di cubiche equianarmoniche e $\infty^{9}$ fasci di cubiche armoniche.

Ora $\dot{e}$ scopo di questo lavoro la determinazione di tutti $i$ fasci le cui cubiche banno un medesimo invariante (o un medesimo modulo) il cui valore sia diverso da uno: la costruzione dei fasci di cubiche di invariante I riesce immediata poichè tali cubiche per un noto teorema di Bertini - devono avere un punto doppio fisso ovvero una parte fissa.

Al risultato richiesto si arriva nel seguente modo.

Si osserva dapprima come a un fascio di cubiche di modulo costante corrisponda

I) S. KAnToR, Premiers fondements pour une théorie des transformations périodiques univoques [Atti della R. Accademia delle Scienze fisiche e matematiche (Società Reale di Napoli), serie II, vol. I (I888), $\mathrm{n}^{\mathrm{0}} 7$ ], pp. 6, 9 .

$\left.{ }^{2}\right)$ A proposito di un fascio più generale di cubiche equianarmoniche che KANToR avrebbe incontrato Cfr. più oltre, $₫ 8$, Nota I 
un fascio di rette e una quartica segata da queste rette secondo quaterne di punti aventi il medesimo birapporto, e viceversa; si costruiscono poi tutte le quartiche siffatte.

Cosi, attraverso la considerazione di queste quartiche, si arriva a riconoscere che i fasci di cubiche a modulo (generico) costante sono $\infty^{8}$ (anzichè $\infty^{3}$ come si era desunto col computo di costanti precedente); sono invece esattamente $\infty^{9} \mathrm{i}$ fasci di cubiche armoniche e $\infty^{\mathrm{II}}$ quelli di cubiche equianarmoniche.

I fasci di cubiche a modulo (generico) costante si dividono in due famiglie distinte, entrambe della medesima dimensione: i fasci della prima famiglia si ottengono prendendo due coniche $K_{1}$ e $K_{2}$ bitangenti e le tangenti comuni $t_{1}$ e $t_{2}$, combinando poi linearmente le due cubiche $K_{1}+t_{1}$ e $K_{2}+t_{2}$; i fasci della seconda famiglia si ottengono invece combinando una cubica degenere in tre rette per un punto con una seconda cubica spezzata in una retta semplice passante per quel punto e in una retta doppia.

I fasci di cubiche equianarmoniche formano un'unica famiglia. Il tipo generale è costituito dal fascio in cui le dodici cubiche dotate di punto doppio si riducono a sei cubiche cuspidate, le cui cuspidi cadon fuori dei nove punti base. Ogni altro fascio di cubiche equianarmoniche rientra in questo come caso particolare: per esempio il fascio costruito da Kantor (che costituisce l'unico fascio di cubiche invarianti per un'omografia ciclica del terz'ordine coi punti uniti giacenti sulle cubiche del fascio) si ottiene facendo divenire a coppie infinitamente vicine le 6 cubiche cuspidate: si ha cosi un fascio in cui vi sono tre cubiche dotate di tacnodo in un punto base del fascio (le tangenti tacnodali non essendo tangenti fisse per le curve del fascio). La costruzione dei fasci di cubiche equianarmoniche, cosi caratterizzati, puó essere ottenuta indirettamente attraverso la quartica segata dalle rette di un fascio secondo quaterne equianarmoniche, di cui è data appunto l'equazione nel $\$ 5$.

I fasci di cubiche armoniche costituiscono tre famiglie distinte, tutte di ugual dimensione 9. I fasci della prima famiglia sono caratterizzati dal possedere quattro cubiche dotate di tacnodo (fuori dei punti base); quelli della seconda famiglia dal possedere due cubiche dotate di tacnodo (fuori dei punti base) e due dotate di cuspide nei punti base (la tangente cuspidale essendo diversa dalla tangente fissa che ivi hanno le curve del fascio); infine $\mathrm{i}$ fasci della terza famiglia sono caratterizzati dal possedere quattro cubiche dotate di cuspide nei punti base (la tangente cuspidale essendo diversa dalla tangente fissa). Ogni altro fascio di cubiche armoniche rientra in uno di questi come caso particolare, quando alcune delle cubiche singolari riescano fra loro infinitamente vicine: vanno segnalati $\mathrm{i}$ fasci di cubiche armoniche, ciascuna delle quali è invariante per una medesima omografia ciclica del quart'ordine, i quali sono $\infty^{8}$ e rientrano come casi particolari nei fasci della seconda famiglia. Anche nel caso delle cubiche armoniche, come per i fasci di cubiche equianarmoniche, la costruzione effettiva si puó ottenere attraverso la corrispondente quartica, di cui appunto sono indicate le equazioni nel $\$ 6$.

Ed ora converrà dare un rapido cenno del contenuto dei paragrafi successivi. 
Nel $\ 2$ si mostra come il problema di determinare $i$ fasci di cubiche a modulo costante si riduca a quello di costruire le quartiche segate dalle rette di un fascio $O$ secondo quaterne le quali abbiano tutte un medesimo birapporto.

I paragrafi 3, 4, 5, 6, 7, sono dedicati appunto alla costruzione di queste quartiche.

Precisamente:

nel $\int 3$ si dànno le proposizioni fondamentali per il resto dell'analisi, cioè si determinano le singolarità della quartica nei punti di contatto con le tangenti uscenti da $O$;

nei paragrafi 4, 5, 6 si costruiscono le quartiche segate secondo quaterne a birapporto costante dalle rette di un fascio il cui sostegno sia esterno alla quartica stessa, nel $n^{\circ} 4$ si esamina il caso che il birapporto abbia un valore generico, nei $n^{i} 5,6$ i casi equianarmonico ed armonico;

nel $\int 7$ si studia il caso, lasciato da parte nei tre paragrafi precedenti, che il centro del fascio, $O$, venga a cadere sulla quartica.

Infine nel $\oint 8$ si ritorna alla considerazione dei fasci di cubiche a modulo costante, che vengon determinati in base ai risulati dei paragrafi precolenti; e, in due note a termine del paragrafo, vengon costruiti direttamente $i$ fasci di cubiche equianarmoniche ed armoniche che sono caratterizzati dall'esistenza di omografie cicliche che lascian ferma ciascuna curva del fascio.

\section{$\oint 2$.}

\section{Riduzione dei fasci di cubiche alla $g_{,}^{\mathrm{I}}$ segata su una quartica da un fascio di raggi.}

Cominciamo col dimostrare che a un fascio di cubiche, aventi un modulo costante $\alpha$, corrisponde un fascio di raggi i quali segano una quartica $f_{4}$ (la quale non puó mai essere degenere in quattro rette per un punto) secondo quaterne di punti il cui birapporto ha il valore $\alpha$, e che viceversa ogni fascio di raggi e quartica siffatti dinno origine a un fascio di cubiche di ugual modulo.

Ciò discende immediatamente dal fatto che ogni fascio di cubiche appartiene a una rete di cubiche con sette punti base la quale definisce un piano doppio razionale con quartica di diramazione (non degenere in quattro rette per un punto), e che, viceversa, come è ben noto, ogni quartica (non degenere in quattro rette per un punto) è sempre quartica di diramazione per un piano doppio siffatto (CLebsch). Ma conviene chiarire la cosa.

Si consideri la rete delle cubiche per 7 punti base:

Poniamo

$$
\lambda_{1} \varphi_{1}\left(x_{1} x_{2} x_{3}\right)+\lambda_{2} \varphi_{2}\left(x_{1} x_{2} x_{3}\right)+\lambda_{3} p_{3}\left(x_{1} x_{2} x_{3}\right)=0 .
$$

$$
\left\{\begin{array}{l}
y_{1}=\varphi_{1}\left(x_{1} x_{2} x_{3}\right) \\
y_{2}=\varphi_{2}\left(x_{1} x_{2} x_{3}\right), \\
y_{3}=\varphi_{3}\left(x_{1} x_{2} x_{3}\right)
\end{array}\right.
$$


resta cosi definita una trasformazione tra il piano $(x)$ e il piano $(y)$ tale che ad ogni punto $Y$ del piano $(y)$ corrispondono due punti $X_{1} X_{2}$ del piano $(x)$, punti che sono definiti come gli altri due punti base del fascio di cubiche omologhe al fascio di rette per $Y$; pertanto le relazioni (I) diconsi definire un piano doppio $(y)$. I due punti $X_{\mathrm{s}}$ e $X_{2}$, omologhi del punto $Y$, coincideranno in un unico punto $X$ se le cubiche del suddetto fascio risultano tangenti fra loro in $X$. Il luogo dei punti $Y$, cui corrispondono due $X$ coincidenti, costituisce la linea di diramazione del piano doppio $(y)$. 亡 facile riconoscere che tale linea è una quartica: infatti ad una retta a del piano $(y)$ corrisponde una cubica $\rho$ della rete, e su questa le altre cubiche della rete segano una involuzione $g_{2}^{\mathrm{r}}$ di coppie di punti la quale ha precisamente quattro punti uniti corrispondenti alle intersezioni della retta $a$ con la linea di diramazione: la quale risulta quindi una quartica. Per di più il birapporto dei quattro punti intersezioni della a con la quartica di diramazione è uguale al modulo della cubica $\varphi$.

Sia infatti $\mu_{0} \bar{p}_{0}+\mu_{1} \bar{\varphi}_{1}$ uno dei fasci, appartenenti alla nostra rete di cubiche, il quale non contenga la $\psi$; la $g_{2}^{1}$ segata dalle curve della rete sulla $\varphi$ è la $g_{2}^{\mathrm{I}}$ segata sulla $\phi$ stessa dal fascio $\mu_{0} \bar{\varphi}_{0}+\mu_{1} \bar{\varphi}_{1}$; alle cubiche di questo corrispondono, proiettivamente, le rette del piano $(y)$ passanti per un punto $O$, e alle cubiche tangenti alla $\varphi$ le rette che congiungono $O$ coi quattro punti comuni alla retta $a$ e alla quartica di diramazione.

Pertanto il birapporto dei suddetti quattro punti è uguale al birapporto dei valori di $\psi_{r}$ che definiscono le cubiche tangenti alla $\varphi ;$ ma questo birapporto, come è ben noto, è uguale a quello delle quattro tangenti condotte alla p da un suo punto qualunque, cioè al modulo della cubica $\rho$; dal che segue l'enunciato.

Con ció risulta chiaro come le cubiche di un fascio, aventi un modulo costante $\alpha$, diano origine alle rette di un fascio seganti, secondo quaterne di birapporto $\alpha$, una quartica $f_{4}$, la quale è quartica di diramazione del piano doppio definito dalle cubiche che passano per sette fra $i$ nove punti base del fascio dato.

Per riconoscere l'inversa, osserviamo anzitutto che il piano doppio definito dalle cubiche per sette punti puó considerarsi come proiezione di una superficie cubica (fatta da un punto della superficie stessa) le cui sezioni piane sono rappresentate dalle cubiche passanti per sei fra i sette punti base suddetti, sicchè la quartica di diramazione $f_{4}$ appare come contorno apparente della superficie cubica vista da un suo punto.

Ciò posto ricordiamo che ogni quartica è contorno apparente di una superficie cubica, la quale si riduce a un cono nel caso che la quartica degeneri in quattro rette per un punto.

Poichè, escluso il cono, ogni superficie cubica è razionale e puó rappresentarsi col sistema delle cubiche passanti per sei punti base, si deduce che ogni quartica non degenere in quattro rette per un punto - é quartica di diramazione per un piano doppio del tipo considerato, e pertanto le rette di un fascio che la seghino secondo quaterne di birapporto $x$ rappresentano cubiche di un fascio aventi il modulo costante $x$. 
E da quanto precede risulta anche come la nostra quartica $f_{4}$ non possa mai ridursi a quattro rette per un punto.

\section{$\$ 3$.}

\section{Proposizioni fondamentali.}

Ora dobbiamo esaminare quando un fascio di rette intersechi una quartica piana $f_{4}=o$ secondo quaterne di punti a birapporto, e quindi ad invariante, fisso.

Osserviamo anzitutto che se i gruppi $G_{4}$ dellla serie lineare $g_{4}^{1}$ segata sulla quartica $f_{4}$ dalle rette per un punto $O$ hanno tutti l'invariante $J=\mathrm{I}$, allora: o il punto $O$ è doppio per la $f_{4}$, o questa ha una parte doppia, e viceversa.

Converrà inoltre notare che il birapporto, e quindi l'invariante $J$, di una quaterna é indeterminato se questa ha punto triplo (o quadruplo) e solo allora: pertanto si ha una $g_{4}^{1}$ i cui gruppi hanno un invariante indeterminato se la $f_{4}$ passa triplamente per $O$, o se contiene una parte contata tre volte.

Esauriti cosi $i$ casi in cui l'invariante $J$ sia indeterminato o abbia il valore fisso $J=\mathrm{I}$, dovremo occuparci esclusivamente delle $g_{+}^{1}$ i cui gruppi $G_{+}$abbiano un invariante fisso ma determinato e diverso da uno: resta cosi escluso che $O$ sia punto doppio (o multiplo) per $f_{4}$ e che la $f_{4}$ stessa possegga parti da contarsi più di una volta.

Per quanto abbiamo osservato sopra $\mathrm{i}$ fasci di cubiche, nella $g_{4}^{\mathrm{r}}$ segata sopra una quartica dalle rette passanti per un punto $O$ vi devono essere 12 gruppi per cui l'invariante ha un valore (generico) assegnato, 6 gruppi armonici $(J=\infty)$ e 4 gruppi equianarmonici $(J=0)$. $\mathrm{Ma}$ - senza ricorrere alla considerazione dei fasci di cubiche questo fatto si puó dedurre direttamente ove si ricordi che l'invariante di una quaterna di punti (il cui birapporto sia uguale ad $\boldsymbol{\alpha}$ ) si può esprimere nella forma

$$
J=\frac{4\left(\mathrm{I}-\alpha+\alpha^{2}\right)^{3}}{(\alpha+\mathrm{I})^{2}(\mathrm{I}-2 \alpha)^{2}(2-x)^{2}}=\frac{4 i^{3}}{j^{2}}
$$

dove $i=o$, e $j=0$ esprimono che la quaterna è armonica o equianarmonica, e dove ${ }_{4} i^{3}-j^{2}=0$ esprime che la quaterna ha un punto doppio.

Infatti, poichè da un punto $O$ qualsiasi del piano vanno alla quartica I 2 tangenti, le quali segano appunto $\mathrm{i}$ gruppi $G_{4}$ dotati di un punto doppio, l'equazione

$$
4 i^{3}-j^{2}=\mathrm{o}, \quad(J=\mathrm{r})
$$

deve esscre del dodicesimo grado nel parametro $\lambda$ che individua il $G_{4}$ entro la $g_{4}^{\mathrm{r}}$, e quindi $i$ e $j$ devono contenere $\lambda$ rispettivamente al sesto e al quarto grado.

Si consideri ora una quartica $f_{+}=0$ tale che il fascio delle rette passanti per un punto $O$ seghi su di essa quaterne di punti aventi tutte un medesimo invariante di valore $K \neq \mathrm{I}$. Anche per questa $g_{+}^{\mathrm{r}}$ particolare vi devono essere dei gruppi il cui inva- 
riante $J$ ha il valore $\mathrm{I}$ : per questi $J$ avendo contemporaneamente il valore $K$ e il valore $I$ deve risultare indeterminato. Ora, poichè il birapporto, e quindi l'invariante, di una quaterna non risulta mai indeterminato se questa non possiede un punto triplo, concludiamo:

I gruppi della $g_{+}^{\mathrm{x}}$ che hanno un punto doppio banno questo almeno come triplo o risultano indeterminati; quest'ultimo caso verificandosi ove la $f_{4}=0$ contenga come parte una retta passante per il punto $O$ sostegno del fascio che sega la $g_{+}^{1}$.

Veniamo ora ad una seconda osservazione che è fondamentale.

Sia $\lambda_{0}$ il valore del parametro $\lambda$, di una $g_{4}^{1}$ qualunque segata dalle rette di un fascio sopra una quartica, corrispondente ad un $G_{4}$ dotato di punto triplo: $\lambda_{n}$ sarà radice delle tre equazioni

$$
\begin{aligned}
& J=0, \quad \text { ciò } \quad i=0 \text {, } \\
& J=\mathrm{I}, \quad \text { cioè } \quad 4 i^{3}-j^{2}=0 \text {, } \\
& J=\infty, \quad \text { cioè } j=0 \text {, }
\end{aligned}
$$

e se $\lambda_{0}$ è radice $r$-pla, con $r \leqslant 6$, di $J=$ I esso è radice multipla secondo $\left[\frac{r}{3}\right]$ per $i=0$ e secondo $\left[\frac{r}{2}\right]$ per $j=0$, indicandosi con $\left[\frac{r}{3}\right]$ e $\left[\begin{array}{c}r \\ 2\end{array}\right]$ il quoziente per eccesso di $r$ diviso rispettivamente per 3 e per 2.

Se $r>6$, ciò è ancora vero se i gruppi della $g_{4}^{\mathrm{x}}$ hanno l'invariante $J$ di un valore costante $K \neq \mathrm{I}$.

Per dimostrare ció occorre escludere che, divisi $4 i^{3}$ e $j^{2}$ per la massima potenza comune di $\lambda-\lambda_{0}$ la loro differenza dia una funzione $F(\lambda)$ che si annulli ancora per $\lambda=\lambda_{0}$, si che l'equazione $J(\lambda)=\mathrm{r}$ abbia $\lambda=\lambda_{0}$ come radice mutipla di un ordine superiore a quello con cui detta radice compare in una (almeno) delle due equazioni

$$
J(\lambda)=0, \quad J(\lambda)=\infty:
$$

a prima vista sembra infatti possibile che $4 i^{3}$ e $j^{2}$ siano divisibili solo per $\left(\lambda-\lambda_{0}\right)^{6}$ mentre sia ancora divisibile per $\lambda-\lambda_{0}$ la differenza

$$
\frac{4 i^{3}}{\left(\lambda-\lambda_{0}\right)^{6}}-\frac{j^{2}}{\left(\lambda-\lambda_{0}\right)^{6}} .
$$

Per escludere questo basta osservare che in tale ipotesi sarebbe

$$
\lim _{\lambda=\lambda_{0}} \frac{4 i^{3}}{j^{2}}=\mathrm{I} .
$$

il che è assurdo supponendosi che per i gruppi della $g_{+}^{1}$ l'invariante $J$ abbia il valore costante $K \neq \mathrm{I}$.

Ricordando che se un'equazione ammette una radice in più di quel che indichi il suo grado essa è identicamente soddisfatta, deduciamo dall'osservazione precedente che:

Se l'equazione $J=\mathrm{I}$ ammette anche una sola radice $\lambda_{0}$ di molteplicità $r$ non di- 
visibile per 3 , la $g_{4}^{\mathrm{I}}$ - qualora i suoi gruppi abbiano un medesimo invariante - risulta costituita tutta di gruppi equianarmonici; e analogamente risulta costituita tutta di gruppi armonici se l'equazione predetta ammette auche una sola radice $\lambda_{0}$ di molteplicita $r$ non divisibile per 2 .

Infatti se $r_{1} r_{2} \ldots r_{s}$ sono le moltepliciti delle radici dell'cquazione $J(\lambda)=\mathrm{I}$, si ha

e quindi

$$
r_{1}+r_{2}+\cdots+r_{s}=12
$$

$$
\left[\frac{r_{1}}{3}\right]+\left[\frac{r_{2}}{3}\right]+\cdots+\left[\frac{r_{s}}{3}\right]>4
$$

se qualcuno degli $r$ non è divisibile per 3 ; e

$$
\left[\frac{r_{1}}{2}\right]+\left[\frac{r_{2}}{2}\right]+\cdots+\left[\frac{r_{3}}{2}\right]>6
$$

se qualcuno degli $r$ non è divisibile per 2 .

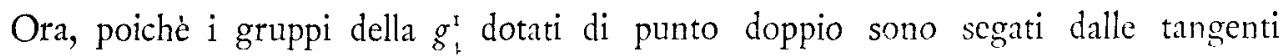
(proprie od improprie) della $f_{4}$ che passano per $O$, possiamo riassumere i risultati ottenuti enunciando $i$ tre teoremi per noi fondamentali:

Teorema I. - Data una quartica $f_{t}$, se una retta uscente da un punto $O$ ba con $f_{4}$ un contatto tripunto e assorbe $r \leqslant 6$ tangenti per 0 , il gruppo da essa segato conta come $\left[\begin{array}{c}r \\ 3\end{array}\right]$ gruppi equianarmonici e $\left[\begin{array}{c}r \\ -2\end{array}\right]$ gruppi armonici, indicandosi con $\left[\begin{array}{c}r \\ 3\end{array}\right] \mathrm{e}$ $\left[\frac{r}{2}\right]$ il quoziente per eccesso di $r$ diviso per 3 c pcr 2: nel caso di $r=6$ tale gruppo conta come 6 fra $i$ gruppi aventi un invariante $K$ prefissato.

Teorema II. - Se per $i$ gruppi di una $g_{4}^{\top}$, segata sopra una quartica $f_{1}$ dalle rette uscenti da un punto $O$, linvariante $I$ ba un valore costante $K \neq \mathrm{I}$, allora le tangenti (proprie od improprie) passanti per $O$ banno con $f_{4}$ un contatto tripunto (almeno), $e$ ciascuna di esse conta - nel gruppo delle i 2 tangenti che da ogni punto vanno ad una quartica - un numero di volte multiplo di 2 se $K \neq 0$, multiplo di 3 se $K \neq \infty$, e multiplo di 6 se contemporaneamente $K \neq$ o e $K \neq \infty$.

Teorema III. - Se la $g_{4}^{1}$ segata sopra una quartica dalle rette di un fascio possiede piu di 4 gruppi equianarmonici, o piu di 6 gruppi armonici o, infine, piu di I2 gruppi il cui invariante $J$ abbia un certo valore $K$ (debitamente computandosi le soluzioni multiple) allora la $g^{\top}$ è costituita di gruppi aventi tutti un medesimo invariante (equianarmonici od armonici nei primi due casi).

I teoremi precedenti permettono di determinare e di costruire tutti i tipi di quartiche sulle quali le rette di un fascio, $O$, seghino una $g_{+}^{1}$ i cui gruppi hanno un birapporto costante: occorre peró sapere-corrispondentemente alle varie singolarità cho puó presentare una quartica - quante tangenti siano assorbite da una retta per $O$ sccondo la natura del suo contatto, che nel nostro caso è scmpre almeno tripunto. 
Diamo qui un elenco di tutti i casi che possono presentarsi nel nostro problema nel quale si suppone che la curva $f_{4}$ non abbia parti multiple e non passi doppiamente per $O$, indicando quale sia, in ciascuno di essi, la molteplicità della tangente: tali molteplicità sono ben note e d'altra parte possono dedursi con facili verifiche analitiche ove si ricordi come le tangenti che da un punto $O$ vanno ad una curva algebrica sono determinate dalle intersezioni di questa con la polare del punto $O$.

Indichiamo con $C$ il punto di contatto, e consideriamo dapprima $i$ due casi in cui $C$ coincide con $O$.

I) Se $C$ è un flesso ordinario, e coincide con $O$, la tangente in $C$ assorbe 3 tangenti.

2) Se $C$ è un flesso del second'ordine (la cui tangente cioè abbia un contatto quadripunto) e coincide con $O$, la tangente in $C$ assorbe 4 tangenti.

In tutti $\mathrm{i}$ casi seguenti $C$ è supposto distinto da $O$.

3) Se $C$ è un flesso ordinario la retta $O C$ assorbe 2 tangenti.

4) Se $C$ è un flesso del second'ordine la retta $O C$ assorbe 3 tangenti.

5) Se $C$ è un punto doppio a tangenti distinte, e una di queste, a contatto bipunto col relativo ramo, coincide con la $O C$, la $O C$ assorbe 3 tangenti.

6) Se $C$ è un punto doppio a tangenti distinte e una di queste, a contatto tripunto col relativo ramo, coincide con la $O C$, la $O C$ assorbe 4 tangenti.

7) Se $C$ è una cuspide ordinaria e $O C$ è la tangente cuspidale, $O C$ assorbe 4 tangenti.

8) Se $C$ è un tacnodo ordinario (due punti doppi infinitamente vicini) e $O C$ è la tangente tacnodale, la $O C$ assorbe 6 tangenti.

9) Se i due rami lineari della quartica che danno il tacnodo precedente vengono a riunirsi in un ramo del second'ordine, la $O C$ assorbe 7 tangenti.

Io) Se in $C$ cadono tre punti doppi infinitamente vicini, essendo la quartica costituita di due rami lineari aventi un contatto tripunto fra loro e bipunto con la $O C$, la $O C$ assorbe 8 tangenti.

I I) Se i due rami di cui sopra vengono a coincidere in un unico ramo del second'ordine, la $O C$ assorbe 9 tangenti.

I2) Se in $C$ cadono quattro punti doppi infinitamente vicini collocati sopra un ramo lineare tangente alla $O C$, la $O C$ assorbe to tangenti.

13) Se $C$ è un punto triplo le cui tre tangenti principali siano distinte fra loro e dalla $O C$, la $O C$ assorbe 6 tangenti.

I4) Se $C$ è un punto triplo e due delle tre tangenti principali coincidono in una sola corrispondente a una cuspide ordinaria, restando la $O C$ distinta dalle tangenti principali, la $O C$ assorbe 7 tangenti.

I 5) Se $C$ è un punto triplo e due delle tre tangenti principali coincidono in una sola corrispondente ad un tacnodo (la $f_{4}$ risulta composta di una cubica che ha un nodo in $C$ e di una delle tangenti principali a detto nodo), restando la $O C$ distinta dalle tangenti principali, $O C$ assorbe 8 tangenti. 
I6) Se $C$ è un punto triplo e le tre tangenti principali vengono a coincidere in una sola, distinta dalla $O C$, dando origine ad un ramo del terz'ordine, la $O C$ assorbe 8 tangenti.

I7) Se $C$ è un punto triplo e le tre tangenti principali vengono a coincidere in una sola, distinta dalla $O C$, dando origine ad un ramo lineare e ad un ramo cuspidale, la $O C$ assorbe 9 tangenti.

I8) Se $C$ è un punto triplo a tangenti distinte, e una di queste - senza far parte della $f_{4}$ - coincide con la $O C$, la $O C$ assorbe 7 tangenti.

I9) Se $C$ è un punto triplo, e due delle tangenti coincidono in una sola diversa dalla $O C$, dando origine ad un ramo cuspidale, e la terza coincide con la $O C$ (senza far parte della $f_{4}$ ), la $O C$ assorbe 8 tangenti.

20) Se $C$ è un punto triplo e due delle tangenti principali coincidono nella retta $O C$, dando origine ad un ramo cuspidale, la $O C$ assorbe 8 tangenti.

2 I) Se $C$ è un punto triplo e due delle tre tangenti principali coincidono dando origine ad un tacnodo, e se la terza tangente coincide con la retta $O C$ (senza far parte della $f_{4}$ ), la $O C$ assorbe 9 tangenti.

22) Se $C$ è un punto triplo, e se le tre tangenti principali coincidono nella $O C$ dando origine ad un ramo del terz'ordine, la $O C$ assorbe 9 tangenti.

23) Se $C$ è un punto quadruplo, e le quattro rette che vengono a formare la $f_{4}$ sono distinte fra loro e dalla $O C$, la $O C$ assorbe 12 tangenti.

24) Se la retta $O C$ si stacca dalla quartica e la cubica residua nion è tangente alla $O C$, questa assorbe 6 tangenti.

25) Se la retta $O C$ si stacca dalla quartica e la cubica residua è tangente (con contatto bipunto) alla $O C$, questa assorbe 7 tangenti.

26) Se la retta $O C$ si stacca dalla quartica e la cubica residua ha in $C$ un contatto tripunto con la $O C$, questa assorbe 8 tangenti.

27) Se la retta $O C$ si stacca dalla quartica e la cubica residua ha in $C$ un punto doppio a tangenti distinte fra loro e dalla $O C$, la $O C$ assorbe 8 tangenti.

28) Se la retta $O C$ si stacca dalla quartica e la cubica residua ha in $C$ un punto doppio a tangenti distinte, e una di queste coincide con la $O C$ (senza far parte della cubica) allora la $O C$ assorbe 9 tangenti.

29) Se la retta $O C$ si stacca dalla quartica e la cubica residua ha in $C$ una cuspide ordinaria e la tangente cuspidale di questa è diversa dalla $O C$, allora la $O C$ assorbe 9 tangenti.

30) Se la retta $O C$ si stacca dalla quartica e la cubica residua ha in $C$ una cuspide ordinaria di cui $O C$ sia la tangente cuspidale, allora la $O C$ assorbe to tangenti.

31) Se la retta $O C$ si stacca dalla quartica e la cubica residua ha in $C$ un tacnodo la cui tangente tacnodale sia distinta dalla $O C$, questa assorbe Io tangenti.

32) Se la retta $O C$ si stacca dalla quartica e la cubica residua ha in $C$ un punto triplo, e le tre rette che la compongono sono distinte fra loro e dalla $O C$, questa assorbe I 2 tangenti. 


\section{$\S 4$.}

\section{Caso del modulo generale.}

Ora possiamo passare alla determinazione e alla costruzione delle $g_{4}^{\mathbf{r}}$, segate sopra una quartica dalle rette di un fascio, i cui gruppi abbiano un birapporto costante: costruiremo prima le $g_{4}^{1}$ prive di punti fissi poi $(\$ 7)$ quelle dotate di un punto fisso: in questo paragrafo esamineremo il caso in cui il valore costante del birapporto sia un valore generico.

Suppongasi adunque che la quartica $f_{4}=0$ sia segata dalle rette passanti per un punto $O$, ad essa esterno, secondo quaterne di punti il cui invariante abbia un valore fisso $K \neq$ o e $\neq \infty$.

In virtù del Teorema II e dell'analisi precedentemente riportata possiamo dire che la $f_{t}=0$ possiede

a) o due tacnodi le cui tangenti tacnodali passano per $O$,

b) o un tacnodo la cui tangente tacnodale passi per $O$ e un punto triplo a tangenti distinte,

c) o due punti tripli a tangenti distinte,

d) o un punto quadruplo.

E evidente come $\mathrm{i}$ casi $b$ ) e $c$ ) non siano possibili, come risulta dalla considerazione della retta che congiunge, nel caso $b$ ), il tacnodo al punto triplo e, nel caso $c$ ), i due punti tripli fra loro.

Restano quindi come soli casi possibili il caso a) e il caso $d$ ).

a) Se la quartica possiede due tacnodi $C_{1}$ e $C_{2}$ le cui tangenti tacnodali passino per $O$, essa si compone - evidentemente - di una coppia di coniche tangenti in $C_{\mathrm{r}}$ e $C_{2}$ alle $O C_{1}$ e $O C_{2}$, e pertanto è agevole costruire una quartica siffatta. Si dimostra facilmente che sopra una tale quartica le rette per $O$ segano quaterne di punti il cui invariante ha un valore costante. Infatti se $K$ è il valore dell'invariante $I$ per una quaterna segata su $f_{4}$ da un certo raggio o passante per $O$ e diverso da $O C_{1}$ e $O C_{2}$, la $g_{4}$ segata dalle rette per $O$ ha 13 gruppi il cui invariante ha il valore $K: 6$ si riuniscono in ciascuno dei gruppi segati dai raggi $O C_{1}, O C_{2}$, e l'ultimo è dato dal raggio o.

Del resto di ciò si puó avere una facile verifica diretta. Si prenda $O$ come punto all'infinito dell'asse $y$, e $C_{1} C_{2}$ come asse $x$ : la quartica avrà allora l'equazione

$$
\left\{y^{2}-\alpha^{2}(x-a)(x-b)\left\{\left\{y^{2}-y^{2}(x-a)(x-b)\right\}=0\right.\right.
$$

essendo $x=a$, e $x=b$ le ascisse di $C_{\mathrm{x}}$ e $C_{2}$. Con ciò le ordinate dei quattro punti segati da una retta per $O$, sono

$$
\begin{aligned}
& y_{1}=\alpha \sqrt{(x-a)(x-b)} \\
& y_{2}=-\alpha \sqrt{(x-a)(x-b)}, \\
& y_{3}=\beta \sqrt{(x-a)(x-b)}, \\
& y_{4}=-\beta \sqrt{(x-a)(x-b)},
\end{aligned}
$$


sicchè il birapporto di questi quattro punti ha il valore costante

$$
\frac{\beta-x}{\beta+x}: \frac{-\beta-x}{-\beta+x} \text {. }
$$

d) Se la quartica possiede un punto quadruplo $C$, riducendosi cosi a 4 rette per $C$, le rette per $O$ segano evidentemente quaterne di punti il cui birapporto ha come valore costante il birapporto delle 4 rette per $C$. Converrà peró ricordare come questa quartica non corrisponda ad un fascio di cubiche di modulo costante (Cfr. $\ 2$ ).

\section{$\int 5$.}

\section{Caso equianarmonico.}

Ci proponiamo ora la determinazione e la costruzione di tutte le quartiche segate dai raggi di un fascio - il cui sostegno $O$ non appartenga alla curva - secondo quaterne tutte equianarmoniche.

A tale oggetto faremo vedere:

a) Presa una cubica arbitraria $\varphi_{3}\left(x_{1} x_{2} x_{j}\right)=0$, non passante per il punto $O=($ oO I $)$, la quartica

$$
f_{4}=\frac{\partial^{2} \varphi_{3}}{\partial x_{3}^{2}} \varphi_{3}-\frac{\mathrm{I}}{2}\left(\frac{\partial \varphi_{3}}{\partial x_{3}}\right)^{2}=0
$$

è segata dalle rette per $O$ secondo quaterne equianarmoniche;

b) Ogni quartica - non passante per $O$ - segata dalle rette per $O$ secondo quaterne equianarmoniche ha un'equazione che rientra come caso particolare nella (I).

In fine del paragrafo determineremo le singolarita che assume la $f_{4}$ al variare della cubica arbitraria $\varphi_{3}$.

Cominciamo dunque col dimostrare che, supposta $p$; generica, la quartica

$$
f_{4}=\frac{\partial^{2} \varphi_{3}}{\partial x_{3}^{2}} \varphi_{3}-\frac{\mathrm{I}}{2}\left(\frac{\partial \varphi_{3}}{\partial x_{3}}\right)^{2}=0
$$

è segata dalle rette per $O$ secondo quaterne equianarmoniche.

Perciò osserviamo anzitutto che la polare del punto $O=(\mathrm{OOI})$ rispetto la $f_{4}$ è la cubica $\varphi_{3}$ : infatti

$$
\frac{\partial f_{4}}{\partial x_{3}}=\frac{\partial^{3} \varphi_{3}}{\partial x_{3}^{3}} \varphi_{3}
$$

e - per ipotesi $-\frac{\partial^{3} \varphi_{3}}{\partial x_{3}^{3}} \neq 0$ in quanto $\varphi_{3}$ non passa per il punto (OoI).

Ciò posto indichiamo con $C_{1} \ldots C_{6}$ i 6 punti d'intersezione della cubica $\varphi_{3}=0$ con la conica (polare di $O$ rispetto la $\varphi_{3}$ ) $\psi_{2}=\frac{\partial \varphi_{3}}{\partial x_{3}}$ : questi punti saranno distinti fra loro supponendosi $\varphi_{3}$ affatto generale. Si vede allora che la quartica $f_{4}$ passa per i punti $C_{1} \ldots C_{6}$, e che questi risultano flessi le cui tangenti sono le rette $O C_{1} \ldots O C_{6}$. 
Infatti la retta $O C_{\mathrm{r}}$ risulta tangente in $C_{1}$ alla cubica $\varphi_{j}$, ed ha pertanto ivi due intersezioni riunite. Si consideri il gruppo $G_{4}$ sezione di $f_{4}$ con la retta $O C_{1}: G_{4}$ contiene $C_{1}$, e il gruppo polare di $O$, rispetto al $G_{4}$, contiene $C_{1}$ contato due volte, essendo $\varphi_{3}$ la polare di $O$ rispetto $f_{4}$ : si deduce - in base a noti teoremi sui gruppi polari - che il $G_{4}$ contiene $C_{1}$ come triplo. Osservando che la retta $O C_{1}$, come le analoghe $O C_{2} \ldots O C_{6}$, non puó assorbire più di due fra le 12 tangenti che da $O$ vanno alla $f_{4}$, si deduce che il punto $C_{1}$ (e cosi gli altri $C_{2} \ldots C_{6}$ ) in cui la retta $O C_{1}$ ha tre intersezioni con la $f_{4}$, è precisamente un flesso (ordinario) di cui $O C_{1}$ è la tangente di flesso.

Che poi le quaterne segate dalle rette per $O$ siano tutte equianarmoniche, risulta subito dai nostri teoremi, osservando che tali sono le 6 quaterne segate dalle rette $O C_{1} \ldots O C_{6}$.

Stabilito cosi che la quartica

$$
f_{4}=\frac{\partial^{2} \varphi_{3}}{\partial x_{3}^{2} \varphi_{3}}-\frac{\mathrm{I}}{2}\left(\frac{\partial \varphi_{3}}{\partial x_{3}}\right)^{2}
$$

è segata secondo quaterne equianarmoniche dalle rette per $O=(\mathrm{OoI})$ quando $\varphi_{;}=0$ è una cubica generica, si puó - in virtù della legge di continuita - concludere che ogni quartica che abbia un'equazione del tipo (I) è effettivamente segata dalle rette per $O$ secondo quaterne equianarmoniche e ciò qualunque sia la cubica $\varphi_{3}$.

Dobbiamo ora dimostrare che - reciprocamente - ogni quartica segata dalle rette per $O=(O O I)$ secondo quaterne equianarmoniche (non aventi peró in $O$ un punto fisso) è rappresentata da un'equazione del tipo

$$
f_{4}=\frac{\partial^{2} \varphi_{3}}{\partial x_{3}^{2}} \varphi_{i}-\frac{\mathrm{I}}{2}\left(\frac{\partial^{2} \varphi_{3}}{\partial x_{3}}\right)^{2} .
$$

Perciò conviene premettere il

Lemma. - Se due quaterne di punti

$$
\begin{aligned}
& \psi_{4}\left(x_{1} x_{2}\right)=a_{0} x_{1}^{4}+a_{1} x_{1}^{3} x_{2}+a_{2} x_{1}^{2} x_{2}^{2}+a_{3} x_{1} x_{2}^{3}+a_{4} x_{2}^{4}=0 \\
& \psi_{4}\left(x_{1} x_{2}\right)=b_{0} x_{1}^{4}+b_{1} x_{1}^{3} x_{2}+b_{2} x_{1}^{2} x_{2}^{2}+b_{3} x_{1} x_{2}^{3}+b_{4} x_{2}^{4}=0
\end{aligned}
$$

sono equianarmoniche e le terne polari di un punto $O=$ (OI) che non appartenga a nessuna di esse coincidono, allora anche le quaterne coincidono.

Infatti, essendo le due terne polari rappresentate da

$$
\begin{aligned}
& a_{1} x_{1}^{3}+2 a_{2} x_{1}^{2} x_{2}+3 a_{3} x_{1} x_{2}^{2}+4 a_{4} x_{2}^{3}=0, \\
& b_{1} x_{1}^{3}+2 b_{2} x_{1}^{2} x_{2}+3 b_{3} x_{1} x_{2}^{2}+4 b_{4} x_{2}^{3}=0,
\end{aligned}
$$

si deduce anzitutto

$$
a_{1}=\rho_{1} b_{1}, \quad a_{2}=\rho_{2} b_{2}, \quad a=\rho b_{3}, \quad a_{4}=\rho_{4}
$$

dove $\rho$ è un coefficiente di proporzionalità diverso da zero. 
Essendo poi le due quaterne equianarmoniche, sarà ${ }^{3}$ )

$$
\begin{aligned}
& a_{2}^{2}-3 a_{1} a_{2}+\mathrm{I} 2 a_{0} a_{4}=0 \\
& b_{2}^{2}-3 b_{1} b_{2}+\mathrm{I} 2 b_{0} b_{4}=0
\end{aligned}
$$

e da queste due uguaglianze, tenuto conto delle quattro precedenti, risulta

$$
a_{\mathrm{o}}=\mathrm{p} b_{\mathrm{o}}
$$

col che resta dimostrato che le due quaterne $\varphi_{4}=0$, e $\psi_{4}=0$ coincidono essendo $\varphi_{4} \equiv \rho \psi_{4}$.

Ciò posto sia $f_{4}=$ o l'equazione di una quartica non passante per il punto $O=$ (OoI) e segata dalle rette per $O$ secondo quaterne equianarmoniche.

Posto

la quartica

$$
\varphi_{3}=\frac{\partial f_{4}}{\partial x_{3}}
$$

$$
f_{4}^{\prime}=\frac{\partial^{2} \varphi_{3}}{\partial x_{3}^{2}} \varphi_{3}-\frac{\mathrm{I}}{2}\left(\frac{\partial \varphi_{3}}{\partial x_{3}}\right)^{2}=0
$$

non passa per $O$ ed è segata dalle rette per $O$ secondo quaterne equianarmoniche: inoltre le terne polari di $O$ rispetto queste quaterne coincidono con le terne polari rispetto le quaterne segate sulla $f_{4}$, essendo date dalle sezioni con la cubica $\varphi_{3}$. In base al lemma precedente si ha dunque che le quaterne segate sulla $f_{+}^{\prime}$ coincidono con quelle segate sulla $f_{4}$, e quindi - risultando le due quartiche identiche - resta dimostrato che la $f_{4}$ ha un'equazione del tipo i).

Rimane cosi stabilito che - fissato un punto $O$ - esistono $\infty 9$ quartiche $f_{4}=0$ segate dalle rette per $O$ secondo quaterne equianarmoniche, dipendendo ciascuna dalla cubica polare di $O$ :

Ora sarà utile, quantunque non necessario, esaminare quali singolarità acquisti la quartica $f_{4}$ col variare della cubica (sua polare) $\varphi_{3}$.

Anzitutto si osservi che ove la $\varphi_{3}$, venisse a passare per il punto $O=(\mathrm{Ool})$, la $f_{4}$ assumerebbe in $O$ un punto quadruplo: infatti in tale ipotesi

è identicamente nulla essendo

$$
\frac{\partial f_{4}}{\partial x_{j}}=\frac{\partial^{3} \varphi_{3}}{\partial x_{3}^{3}} \varphi_{3}
$$

$$
\frac{\partial^{3} p_{3}}{\partial x_{3}^{3}}=0
$$

ed è noto che, condizione necessaria e sufficiente perchè svanisca la polare di un punto $O$ rispetto ad una curva d'ordine $n$ c che il punto $O$ sia $n$-plo per la curva stessa. D'altra parte che $O$ sia quadruplo per la $f_{4}$ puó vedersi anche con una verifica analitica diretta.

3) Cfr. F. Enriques e O. Chisini, Lezioni sulla teoria geometrica delle equazioni, vol. I (Bologna, Zanichelli, 1915), p. 29. 
Escluso dunque che la cubica $\varphi_{3}$, venga a passare per il punto $O$, per esaminare cosa accada della quartica $f_{+}$al variare della $\varphi_{j}$, conviene distinguere $\mathrm{i}$ due generi di particolarizzazione che si hanno secondo che varia la posizione di $O$ rispetto la $\varphi_{3}$, o varia la $\rho_{3}$ acquistando un punto doppio o altre singolarità.

I) Suppongasi che, variando la posizione relativa della cubica $\varphi_{3}=0$ e del punto $O$, questo venga a cadere sopra una tangente di flesso della $\varphi_{i}$, la quale tuttavia resti una cubica generale. In tale ipotesi due punti $C_{1}$ e $C_{2}$, intersezioni della cubica $\varphi_{3}$ con la conica $\psi_{2}$ - polare di $O$ rispetto alla $\psi_{3}$ - vengono a coincidere in un unico punto $C$, e pertanto vengono a coincidere nella $O C$ le due rette $O C_{1}$ e $O C_{2}$ che erano tangenti di flesso per la quartica $f_{4}$. Quale sard il comportamento della $f_{4}$ nel punto $C$ ? Per determinarlo osserviamo che la retta $O C$ ha un contatto tripunto con la cubica $\varphi_{3}$ (che è la polare di $O$ rispetto la $f_{4}$ ) e quindi ha - pure in $C$ - quattro intersezioni riunite con la $f_{4}$ : inoltre la $O C$ assorbe quattro tangenti e quindi, come risulta dall'analisi riprodotta nell'elenco del $n^{\circ} 3, C$ è un nodo di cui un ramo ha come tangente di flesso la $O C$.

2) Suppongasi ora che $-O$ restando generico - la cubica $\varphi_{3}$ vari acquistando un punto doppio $C$ : anche in questo caso due punti, $C_{1}$ e $C_{2}$, intersezioni di $\varphi_{;}$con la conica polare $\psi_{2}$, vengono a coincidere nel punto $C$, e le due rette $O C_{1}, O C_{2}$, tangenti di flesso per la $f_{4}$, si riuniscono nella retta $O C$. Questa retta ha in $C$ due intersezioni riunite con la $\rho_{3}$ e quindi tre con la $f_{4}$; d'altra parte essa assorbe 4 tangenti per $O$ : pertanto, dall'esame del quadro dato nel $n^{\circ} 3$ si deduce che il punto $C$ è - per $f_{4}$ - una cuspide di cui $O C$ è la tangente cuspidale.

3) Suppongasi che - essendo la $\varphi_{;}$dotata di un nodo $C$ - il punto $O$ cada su una delle tangenti principali in $C$ : in questo caso la retta $O C$ ha con la $f_{4}$ quattro intersezioni riunite in $C$, e assorbe 6 tangenti: si deduce, come nei due casi precedenti, che $C$ è per $f_{4}$ un tacnodo di cui $O C$ è la tangente tacnodale.

4) Suppongasi che - restando $O$ in posizione generica - la cubica $\varphi$, acquisti una cuspide $C$ : in tale ipotesi la retta $O C$ viene ad avere tre intersezioni riunite con la $f_{4}$ e assorbe 6 tangenti: si deduce che $C$ è per $f_{4}$ un punto triplo le cui tre tangenti sono distinte fra loro e dalla $O C$.

5) Suppongasi che - la cubica $\varphi_{3}$ essendo dotata di una cuspide $C$-il punto $O$ venga a cadere sulla tangente cuspidale: in tale ipotesi la tetta $O C$ ha con $f_{4}$ quattro intersezioni riunite ed assorbe 8 tangenti.

Tenuto conto che questo caso è un caso limite del precedente, si ha anzitutto che $C$ deve essere un punto triplo: dall'esame dell'clenco del paragrafo 3 si ricava poi che:

o $C$ è un punto triplo di cui due delle tangenti coincidono in una sola diversa dalla $O C$ dando origine ad un ramo cuspidale e la terza coincide con la $O C$ (caso 19); oppure $C$ è un punto triplo e due delle tangenti principali coincidono nella retta $O C$ dando origine ad un ramo cuspidale (caso 20).

Ora è facile riconoscere che effettivamente ha luogo il secondo di questi due casi 
potendosi dimostrare l'assurdità del primo. Suppongasi infatii che esso si verifichi: segando la $f_{4}$ con una retta $o^{\prime}$ passante per $O$ e prossima alla $O C$, il ramo tangente ad $O C$ è intersecato in due punti $A_{1} A_{2}$ e il ramo cuspidale in altri due $B_{1} B_{2}$ tali che - assunto come infinitesimo unità l'angolo della retta $O^{\prime}$ con la $O C$ - la distanza $B_{1} B_{2}$ è infinitesima d'ordine $\frac{3}{2}$, mentre la $A_{1} A_{2}$ è infinitesina d'ordine $\frac{1}{2}$ conse anche la $A_{1} B_{2}$ e la $B_{1} A_{2}$ : pertanto il birapporto

$$
\left(A_{1} B_{\mathrm{r}} A_{2} B_{2}\right)=\frac{A_{1} A_{2}}{B_{1} A_{2}}: \frac{A_{1} B_{2}}{B_{1} B_{2}}=\frac{A_{1} A_{2} \cdot B_{1} B_{2}}{B_{1} A_{2} \cdot A_{1} B_{2}}
$$

é infinitesimo, e quindi la $g_{4}^{\mathrm{I}}$ segata dalle rette per $O$ non puó esser costituita tutta da gruppi equianarmonici per $\mathrm{i}$ quali il birapporto ha $\mathrm{i}$ due valori

$$
-e^{\frac{2 \pi i}{3}}, \quad-e^{\frac{4 \pi i}{3}}
$$

6) Suppongasi che la $p_{3}$ acquisti un tacnodo $C$, restando $O$ fuori dalla tangente tacnodale: la retta $O C$ ha tre intersezioni con la $f_{+}$e assorbe 8 tangenti: si deduce che la $f_{4}$ ha in $C$ un punto triplo in cui le tre tangenti coincidono in una (diversa dalla $O C$ ) dando origine ad un unico ramo del terz'ordine.

7) Suppongasi che, la $\varphi_{3}$ possedendo un tacnodo $C$, il punto $O$ venga a cadere sulla tangente tacnodale, e quindi sulla cubica $\rho_{;}$: come si c già notato, in tale ipotesi la $f_{4}$ acquista un punto quadruplo in $O$, e una delle rette che la compongono i la tangente tacnodale.

8) Suppongasi in fine che la $q$; abbia un punto triplo $C$ : la $O C$ viene ad avere con la $f_{4}$ quattro intersezioni riunite e assorbe 12 tangenti: si deduce che $C$ è per $f_{4}$ un punto quadruplo.

$\$ 6$.

\section{Caso armonico.}

La determinazione delle quartiche $f_{+}=0$ segate dalle rette di un fascio, il cui sostegno $O$ sia esterno alla quartica, secondo quaterne tutte armoniche, riesce piuttosto complicata poichè vi è non una ma tre famiglie distinte di quartiche siffatte.

Occorre pertanto esaminare e costruire isolatamente i singoli casi particolari possibili, e per procedere nel modo pit semplice conviene trattare dapprima le quartiche irriducibili e poi quelle riducibili.

Suppongasi adunque dapprima che la quartica $f_{4}=0$ sia irriducibile: dall'csame istituito nel $n^{\circ} 3$ risulta - in base al nostro teorema fondamentale - che le singolarità presentate da una quartica, segata dalle rette per $O$ secondo quaterne armoniche, sono solo

a) flesso del second'ordine la cui tangente passi per $O$ (caso 4); 
b.) punto doppio a tangenti distinte di cui una, a contatto bipunto col relativo ramo, passi per $O$ (caso 5 );

c) tacnodo la cui tangente tacnodale passi per $O$ (caso 8);

d) punto triplo le cui tangenti principali siano distinte fra loro e nessuna di esse passi per $O$ (caso I 3 );

e) punto triplo le cui tre tangenti principali coincidono in una sola, passante per $O$, dando origine ad un ramo del terz'ordine (caso 22).

Si esclude infatti anzitutto il caso I I che assorbe 9 tangenti, cioè l'esistenza di un punto doppio $C$, con due punti doppi infinitaments vicini su un ramo lineare tangente alla $O C$, e origine di un ramo del second'ordine per la $f_{4}$, nel modo che segue. E chiaro infatti come la $f_{4}$ non puó avere un altro punto doppio distinto da $O C$, perchè in tal caso il ramo del second'ordine che ha l'origine in $C$ si spezzerebbe in due rami lineari; quindi le altre tre tangenti uscenti da $O$ dovrebbero riunirsi in una tangente con contatto quadripunto in un punto $F$, flesso del second'ordine. Si riconosce allora come la quartica $f_{4}$ apparterrebbe al fascio determinato dalla retta $C F$ contata quattro volte, e dalla quaterna di rette $3 O C+O F$, passando per i loro 16 punti comuni. Pertanto $f_{f}$ doviebbe avere in $O$ un punto triplo, contro l'ipotesi.

Si esclude inoltre che la $f_{4}$ abbia un punto triplo $C$, in cui le tre tangenti principali vengano a coincidere in una sola distinta dalla $O C$, dando origine a un ramo lincare e ad un ramo cuspidale (caso 17 ), e cosi pure che abbia un punto triplo $C$ di cui una tangente principale passi per $O$ e le altre due coincidano dando origine ad un tacnodo (caso 2 I).

A tale oggetto si consideri la quaterna segata da una retta $o^{\prime}$, passante per $O$, e prossima alla $O C$ : si riconosce, con semplici considerazioni di infinitesimi, che tali quaterne - convenientemente ordinate - hanno un birapporto infinitesimo, il che è contradittorio con l'ipotesi che le quaterne segate dalle rette per $O$ siano tutte armoniche, ció che il loro birapporto abbia i valori $-\mathrm{I}, 2, \frac{\mathrm{I}}{2}$.

Indicheremo con $A, B, \ldots, E$ un punto della quartica del tipo $a), b), \ldots, e)$ : una quartica irriducibile puó avere:

I) 4 punti $A$,

2) 3 punti $A$ e I punto $B$,

3) 2 punti $A$ e 2 punti $B$,

4) I punto $A$ e 3 punti $B$,

5) I punto $C$ e 2 punti $A$,

6) I punto $C$, I punto $A$, e I punto $B$,

7) I punto $D$ e 2 punti $A$,

8) I punto $E$ e I punto $A$.

Ora noi daremo la costruzione delle quartiche corrispondenti ai casi effettivamente possibili e dimostreremo l'impossibilità dei rimanenti.

I) La quartica $f_{4}$ possegga 4 flessi del second'ordine $A_{1} \ldots A_{4}$ le cui tangenti passino per $O$. 
La retta $a$, polare di $O$ (rispetto alla $f_{4}$ ) passa per questi quattro punti, che risultano cosi allineati: pertanto la quartica appartiene al fascio determinato dalla retta $a$ contata quattro volte e dalla quaterna di rette costituita dalle tangenti di flesso nei punti $A$.

$\mathrm{Ne}$ viene per la $f_{4}$ un'equazione del tipo

dove

$$
f_{4}=a_{x}^{4}+\lambda b_{x} c_{x} d_{x} e_{x}
$$

$$
b_{x}=0, \quad c_{x}=0, \quad d_{x}=0, \quad e_{x}=0
$$

sono l'equazioni di quattro rette passanti per un punto $O$, e

$$
a_{x}=0
$$

quella di una quarta retta non passante per $O$.

$\mathrm{Si}$ riconosce facilmente che qualunque quartica del fascio precedente è effettivamente segata dalle rette per $O$ secondo quaterne armoniche: infatti vi sono 4 quaterne (quelle segate dalle rette $b_{x}=0, \ldots, c_{x}=0$ che assorbono 3 tangenti) ciascuna delle quali, in virtù del Teorema I, conta per 2 quaterne armoniche, e quindi, per il Teorema III, tutta la $g_{4}^{I}$ è costituita di quaterne armoniche.

Ció si puó dedurre anche direttamente osservando che la $f_{4}$ resta invariata per un'omologia ciclica del quart'ordine che ha $O$ come centro e $a$ come asse, ove si ricordi che - sopra una retta - ogni ciclo di una proiettività ciclica del quart'ordine costituisce una quaterna armonica.

2) Non è possibile che la quartica $f_{4}$ possegga 3 flessi del second'ordine $A_{1}, A_{2}, A_{3}$, le cui tangenti passino per $O$, e un nodo $B$ di cui un ramo sia tangente alla $O B$. Infatti la considerazione della retta polare di $O$ mostra che i punti $A_{1}, A_{2}, A_{3}$ si trovano sopra una stessa retta $a$, la quale viene a far parte della conica $\Psi_{2}$ polare di $O$, che risulta cosi costituita dalla retta a contata due volte. Pertanto il punto $B$, che appartiene alla conica polare, si trova anch'esso sulla retta polare $a$; si deduce che la retta $O B$ ha in $B_{4}$ intersezioni riunite con la $f_{4}$, e quindi $B$ è un flesso del second'ordine la cui tangente passa per $O$ e non un punto doppio quale si era supposto.

3) La $f_{4}$ possegga due flessi del second'ordine $A_{1}, A_{2}$ e due nodi $B_{\mathrm{s}}, B_{2}$, le rette $O A_{1}, O A_{2}$ essendo tangenti di flesso e le $O B_{1}, O B_{2}$ tangenti (a contatto bipunto) di un ramo del nodo. La considerazione della conica polare di $O$ mostra che $B_{1}$ e $B_{2}$ sono sopra una conica che è tangente in $A_{1}$ e $A_{2}$ alle rette $O A_{1}$ e $O A_{2}$.

Pertanto la quartica più generale di questo tipo si costruisce nel modo seguente. Si assumano ad arbitrio i punti $O, A_{1}, A_{2}, B_{1}$ e si consideri la conica $\psi_{2}$ passante per $B_{1}$ e tangente in $A_{1}, A_{2}$ rispettivamente alle rette $O A_{1}, O A_{2}$.

Sia $B_{2}$ un punto qualunque di questa conica $\psi_{2}$. Si costruisca la quartica che ha in $A_{1}$ e $A_{2}$ come tangenti a contatto quadripunto le due rette $O A_{1}$ e $O A_{2}$, che ha in $B_{1}$ un punto doppio, di cui un ramo tangente alla $O B_{1}$, e che in fine $\dot{e}$ tangente in $B_{2}$ alla $O B_{2}$. Poiché le condizioni imposte si traducono in 14 equazioni lineari nei coefficienti incogniti della quartica, esiste certo (almeno) una quartica $f_{4}^{\prime}$ che le sod- 
disfi. Osserviamo che la retta $O B_{2}$ viene ad avere 3 intersezioui riunite con la $f_{4}^{\prime}$ : infatti per $B_{2}$ passa la cubica e la conica polare di $O$ rispetto la $f_{4}^{\prime}$. Da ciò risulta che la $g_{4}^{\mathbf{I}}$, segata dalle rette per $O$ sopra la $f_{4}^{\prime}$, è costituita da gruppi armonici avendo 7 gruppi siffatti: quelli segati dalle rette $O A_{1}, O A_{2}, O B_{1}$, che contano ciascuno per due (Teorema I), e quello segato dalla retta $O B_{2}$ che conta almeno una volta. Da ció si deduce che la retta $O B_{2}$, che conta almeno due volte nel numero delle tangenti alla $f_{4}$ uscenti da $O$, ne conta effettivamente tre, e quindi il punto $B_{2}$ risulta un punto doppio di cui un ramo è tangente alla $O B_{2}$ : si esclude infatti che $B_{2}$ possa essere un flesso del second'ordine, in base all'osservazione fatta precedentemente della non esistenza di una quartica $f_{4}$ dotata di tre flessi del second'ordine $A_{1}, A_{2}, A_{3}$, e di un nodo $B$.

Si noti che da quanto sopra è detto risulta anche l'unicita della quartica soddisfacente alle condizioni proposte: infatti se $f_{4}$ e $f_{4}^{\prime}$ sono due quartiche siffatte esse hanno in comune 8 punti ( 4 in $A_{1}, 4$ in $A_{2}, 5$ in $B_{1}, 5$ in $B_{2}$ ) e pertanto coincidono.

4) Non puó accadere che la quartica $f_{4}$ possegga un flesso del second'ordine $A$ e tre punti doppi $B_{1} B_{2} B_{3}$. Dedurremo ció dall'analisi - che istituireno più avanti delle quartiche dotate di quattro punti doppi $B_{1} \ldots B_{4}$ (e però riducibili in due coniche); per ora continuiamo l'analisi delle $f_{4}$ irriducibili.

5) Una quartica $f_{4}$ la quale possegga un tacnodo $C$, la cui tangente tacnodale passi per $O$, e due flessi del second'ordine $A_{1}, A_{2}$, le cui tangenti passino pure per $O$, appare come caso particolare delle quartiche esaminate in I).

Infatti la considerazione della retta $a$, polare di $O$ rispetto la quartica $f_{4}$, mostra che i tre punti $C, A_{1}, A_{2}$ sono allineati: si deduce cosi per la $f_{4}$ un'equazione del tipo

$$
f_{4}=a_{x}^{4}+\lambda b_{x} c_{x} d_{x}^{2}
$$

che è appunto un caso particolare della

$$
f_{4}=a_{x}^{4}+\lambda b_{x} c_{x} d_{x} e_{x}
$$

E inutile dire che ogni quartica

$$
f_{4}=a_{x}^{4}+\lambda b_{x} c_{x} \cdot d_{x}^{2}
$$

ha effettivamente un tacnodo nel punto $C$, intersezione delle rette $a_{x}=\mathrm{o}$ e $d_{x}=\mathrm{o}$, e che è segata dalle rette per $O$ secondo quaterne tutte armoniche.

6) Non è possibile che la quartica $f_{4}$ possegga un tacnodo $C$ un flesso $A$ e un punto doppio $B$. Ciò si esclude nello stesso modo con cui si è escluso il caso 2).

Si ricordi che la polare di un punto $O$ che si trova sopra la tangente tacnodale di una curva qualsiasi $C_{n}$ possiede come triplo il tacnodo di $C_{n}$, come risulta da una facile verifica analitica: pertanto la cubica polare di $O$ (rispetto la nostra quartica $f_{4}$ ) ha $C$ come triplo, e la conica polare di $O$ ha $C$ come doppio: ma tale conica polare deve passare anche per $A$ ed essere ivi tangente alla $O A$, quindi essa si riduce alla retta $C A$ contata due volte. Pertanto il punto $B$ viene ad essere allineato con $A C$, e la retta $O B$ viene ad avere due intersezioni con la conica polare di $O$ e quindi quattro 
con la $f_{4}$ : si deduce che $C$ non è un nodo ma un flesso del second'ordine la cui tangente passa per $O$.

7) La quartica $f_{+}$possegga un punto triplo $D$ a tangenti distinte fra loro e dalla $O D$, e due flessi del second'ordine $A_{1}, A_{2}$, le cui tangenti passino per $O$.

Poichè un punto triplo impone solo 6 condizioni e 4 ne impone un flesso del second'ordine di cui sia data anche la tangente, cosi si possono assumere ad arbitrio i punti $O, D, A_{1}, A_{2}$, col che resta determinata linearmente una quartica $f_{1}$ : infatti se $f_{4}$ e e $f_{4}^{\prime}$ sono due quartiche aventi un punto triplo in $D$ e due flessi del second'ordine nei due punti $A_{1}$ e $A_{2}$ avendo ivi per tangenti le rette $O A_{1}$ c $O A_{2}$, csse hanno i7 punti comuni e quindi coincidono. Scegliendo convenientemente le coordinate si ha per la $f_{4}$ un'equazione abbastanza semplice: posto

si ha

$$
D \equiv(\mathrm{OOI}), \quad A_{\mathrm{I}} \equiv \text { (OIO) }, \quad A_{2} \equiv(\mathrm{OOI}), \quad O \equiv(\mathrm{III})
$$

$$
f_{4}=x_{1}^{3} x_{3}+x_{1}^{2} x_{2} x_{3}+x_{1} x_{2}^{2} x_{3}+x_{2}^{3} x_{3}-x_{1}^{3} x_{2}-x_{1}^{2} x_{2}^{2}-x_{1} x_{2}^{3}=0 .
$$

Sull'equazione della $f_{4}$ si puó verificare direttamente che le rette per $O$ segano effettivamente quaterne tutte armoniche, ma ció risulta a priori, in base ai nostri teoremi fondamentali, osservando che la retta $O D$ sega un gruppo che conta come 3 gruppi armonici, e le rette $O A_{1}, O A_{2}$ segano gruppi ciascuno dei quali conta come 2 gruppi armonici.

In virtù dell'unicità della quartica soddisfacente alle precedenti condizioni, si riconosce facilmente che tale quartica rientra come caso particolare in quelle determinate in 3): infatti se nella costruzione ivi indicata si assume $B_{2}$ infinitamente vicino al punto $B_{1}$, tale punto - assorbendo 6 delle tangenti uscenti da $O$, ed essendo intersezione semplice della $O B_{\mathrm{r}}$ con la conica polare di $O$ rispetto la $f_{4}$ - risulta un punto triplo.

8) La $f_{4}$ possegga un punto triplo $E$, le cui tre tangenti principali coincidano nella $O E$ dando origine ad un ramo del terz'ordine, e un flesso del second'ordine $A$, la cui tangente passi per $O$.

Si riconosce facilmente che questo caso rientra come caso particolare in quello esaminato in $I$ ).

Infatti la quartica risulta evidentemente appartenere al fascio determinato dalla retta $a=E A$ contata quattro volte e dalla quartica composta della retta $O E$ contata tre volte e della $O A$ : si deduce per la quartica un'equazione del tipo

$$
f_{4}=a_{x}^{4}+\lambda b_{x} c_{x}^{3}
$$

ed è inutile osservare che effettivamente le rette per $O$ segano quaterne armoniche.

Cosi è esaurita l'analisi e la costruzione delle quartiche irriducibili segate dalle rette di un fascio, il cui sostegno $O$ sia esterno alla curva, secondo quaterne di punti tutte armoniche. Veniamo ora alle quartiche riducibili che supporremo sempre - come più volte si è detto - prive di parti multiple e non passanti per $O$.

Converrà osservare anzitutto che se la quartica $f_{4}$ contiene una retta $r$, deve con- 
tenerne una seconda $r^{\prime}$. Infatti se $R$ è l'intersezione della $r$ con un raggio generico $o$ del fascio $O$, il punto $R^{\prime}$ che con $R$ separa armonicamente gli altri due punti della quaterna segata da o, è unico, e quindi - non potendo mai coincidere con $O$ - descrive, al variare di o, una retta $r^{\prime}$,

Ció posto gli unici tipi possibili di quartiche riducibili segate dalle rette per $O$ secondo quaterne armoniche sono:

I) Quartica degenere in una coppia di coniche, $K_{1}$ e $K_{2}$;

2) Quartica degenere in una coppia di rette, $r$ e $r^{\prime}$ e in una conica $K$;

3) Quartica degenere in quattro rette.

I) Cominciamo con l'esaminare il primo tipo di quartiche riducibili. Supponiamo

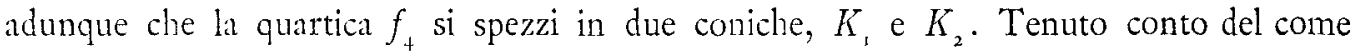
debbono riunirsi le 12 tangenti uscenti da $O$ perchè i gruppi segati dalle rette per esso risultino tutti armonici, si vede facilmente che questo tipo presenta tre casi distinti:

a) Le due coniche $K_{1}$ e $K_{2}$ sono fra loro bitangenti dando origine a due tacnodi, $C_{1}$ e $C_{2}$ le cui tangenti tacnodali passano per $O$. Questo risulta evidentemente un caso particolare delle quartiche segate dalle rette per $O$ secondo quaterne il cui invariante ha un valore fisso $K$ generico, e anche come caso particolare delle quartiche I) studiate nel principio di questo paragrafo.

Si deduce per la quartica $f_{4}$ un'equazione del tipo

$$
f_{4}=a_{x}^{4}+\lambda b_{x}^{2} c_{x}^{2}
$$

essendo $O$ il punto comune alle rette $b_{x}=\mathrm{o}$ e $c_{x}=0$, e $C_{x}$ e $C_{2}$ le intersezioni di queste rette con la $a_{x}=0$.

b) Le coniche $K_{1}$ e $K_{2}$ sono fra loro bitangenti in un punto $C_{\mathrm{r}}$ e s'intersecano ulteriormente in due punti, $B_{1}$ e $B_{2}$, la $O C_{1}$ essendo tangente comune, la $O B_{1}$ e la $O B_{2}$ tangenti rispettivamente alla $K_{1}$ e $K_{2}$.

Una quartica siffatta si puó ottenere evidentemente prendendo ad arbitrio i quattro punti, $O, C_{1}, B_{1}, B_{2}$ : che essa sia segata dalle rette per $O$ secondo quaterne tutte armoniche risulta dall'esistenza di 7 quaterne siffatte (tre segate dalla retta $O C_{1}$ e due da ciascuna delle altre $O B_{1}$ e $O B_{2}$ ).

c) Le coniche $K_{1}$ e $K_{2}$ s'intersecano in quattro punti distinti, $B_{1}, B_{2}, B_{3}, B_{4}$, ciascuna delle rette, $O B_{1}, O B_{2}, O B_{3}, O B_{4}$, essendo tangente ad una delle coniche $\left(O B_{1}\right.$ e $O B_{2}$ alla $K_{1}, O B_{3}$ e $O B_{4}$ alla $\left.K_{2}\right)$.

Per venire alla costruzione delle quartiche di questo tipo premettiamo il

Lemma. - Dato un fascio di coniche passanti per due punti assegnati $B_{2}, B_{;} e$ tangenti in un punto pure assegnato $B_{1}$ ad una retta fissa $O B_{1}$, il luogo dei punti $B_{4}$, punti di contatto delle coniche del fascio con le rette per $O$, è la conica $K_{1}$ che passa per $B_{1}, B_{2}, B_{3}$ ed è tangente in $B_{2}$ e $B_{3}$ alle rette $O B_{2}, O B_{3}$.

Per ottenere la dimostrazione del nostro lemma osserviamo che, in generale, dato un fascio di coniche, i cui punti base siano distinti, il punto $X$ di contatto di queste coniche con le rette di un fascio $O$ descrive una cubica passante per $O$ : vi sono in- 
fatti due coniche tangenti ad una retta generica per $O$ ed una tangente in $O$ ad una retta del fascio. Ora se due punti base del fascio vengono a trovarsi sopra una retta a passante per $O$, questa si stacca dalla cubica e il luogo descritto dai punti $X$ è una conica. Dunque nel nostro caso il luogo descritto dal punto $B_{4}$ è una conica: si vede subito che questa passa per $B_{1}, B_{2}, B_{;}$; che essa poi sia tangente in $B_{2}$ (e cosi in $B_{3}$ ) alla $O B_{2}$ lo si deduce dal fatto che vi è una sola conica del fascio tangente alla $O B_{2}$.

Ciò posto il tipo generale di quartica $f_{4}$, degenere in una coppia di coniche $\mathrm{i}$ cui quattro punti comuni $B_{1} \ldots B_{4}$ siano distinti, la quale sia segata dalle rette di un fascio $O$ secondo quaterne tutte armoniche, si ottiene facilmente come segue.

$\mathrm{Si}$ assumano ad arbitrio il punto $O$ e i tre punti $B_{1}, B_{2}, B_{3}$. Ciò posto si costruisca la conica $K_{2}$ che passa per $B_{1}, B_{2}, B_{3}$ ed è tangente in $B_{2}$ e $B_{3}$ alle $O B_{2}$, $O B_{;}$; poi si prenda una qualunque conica, $K_{1}$, che passa per $B_{2}, B_{3}$ ed è tangente in $B_{1}$ alla $O B_{1}$ : questa interseca la $K_{2}$ in un quarto punto $B_{4}$ e la retta $O B_{4}$ risulta, per il lemma stabilito innanzi, tangente alla $K_{\mathrm{i}}$.

Pertanto la quartica $f_{4}$ composta della $K_{t}+K_{2}$ possiede quattro punti doppi, $B_{1}, B_{2}, B_{3}, B_{4}$, di cui una tangente principale passa per $O$ : si deduce che le rette per $O$ segano su questa quartica quaterne tutte armoniche, contando due volte come tali quelle segate dalle rette $O B_{1} \ldots O B_{4}$.

In base a quanto si è ora visto possiamo escludere l'esistenza di una quartica $f_{4}-$ segata dalle rette per $O$ secondo quaterne armoniche - dotata di un flesso del second'ordine $A$, e di tre punti doppi, $B_{1}, B_{2}, B_{i}$, essendo $O A$ tangente di flesso e $O B_{1}$, $O B_{2}, O B_{3}$, tangenti principali. [Questo é il tipo 4) delle quartiche irriducibili esaninate nella prima parte di questo paragrafo e di cui ivi avevamo annunciato l'impossibilità].

Perciò occorre notare the presi ad arbitrio i punti $O, B_{1}, B_{2}, B_{\text {; }}$, esistono 6 quartiche $f_{4}$ del tipo precedente, cioè degeneri in due coniche, il cui ultimo punto doppio $B_{4}$ giaccia sopra una retta asscgnata passante per $O$ : infatti i tre punti, $B_{1}, B_{2}, B_{3}$, definiscono 3 coniche $K_{2}$, dipendentemente dalla scelta della coppia di punti $B_{2}$ e $B_{3}$ in cui la $K_{2}$ è presa tangente allc rette $O B_{2}, O B_{3}$.

Occorre inoltre ricordare che in una rete di quartiche - quale è quella delle quartiche aventi come doppi i punti $B_{1}, B_{2}, B_{3}$ e come tangenti principali le $O B_{1}, O B_{2}$, $O B_{;}$- ve ne sono 6 che hanro un contatto tripunto con una retta $a$ generica del piano. Questa nota proposizione puó desumersi mediante una facile applicazione del principio di corrispondenza: preso un punto $A$, della retta $a$, esistono 4 punti $A^{\prime}$ in cui è tangente alla $a$ una quartica della rete che passi per $A$, viceversa ogni punto $A^{\prime}$ sorge in tal modo da 2 punti $A$; pertanto vi sono 6 punti della retta $a$ per cui $A$ e $A^{\prime}$ coincidono, cioé punti in cui una quartica della rete ha con $a$ un contatto tripunto.

Ora, da quanto è stato sopra osservato risulta che le 6 quartiche aventi con una retta $a$ - passante per $O$ - un contatto tripunto hanno su $a$ un punto doppio per cui $a$ è tangente principale. Resta cosi escluso che esista una quartica $f_{4}$ la quale abbia tre punti doppi, $B_{1}, B_{2}, B_{3}$ (di cui una tangente principale passi per $O$ ) e un flesso del 
second'ordine $A$ la cui tangente $a$ passi pure per $O$. Ma, per rendere affatto rigorosa la deduzione precedente, occorre aggiungere che è assurda l'esistenza di una retta $a$ (diversa dalle $O B_{1}, O B_{2}, O B_{3}$ ) su cui esistano infiniti punti di contatto tripunto per una quartica della rete senza che la $a$ ne faccia parte: infatti solo una retta siffatta potrebbe essere la tangente del flesso di second'ordine $A$.

Mostriamo dunque l'assurdità di una tale ipotesi. Sia $\overline{f_{4}}$ una quartica (generica) della rete che ha un contatto tripunto con la $a$ in un punto $\bar{A}$ : questa quartica é segata dalle rette per $O$ secondo quaterne armoniche (ve ne sono 7 di tali) e quindi il punto $\bar{A}$, non essendo per ipotesi un nodo, deve essere un punto in cui il contatto è effettivamente quadripunto e non tripunto. $\mathrm{Ma}$, come abbiamo gid osservato, vi sono 6 quartiche (composte di due coniche $K_{1}$ e $K_{2}$ ) che hanno su $a$ un punto doppio: una qualunque di queste dovrebbe avere (come una $\bar{f}_{4}$ generica) un contatto quadripunto, il che è assurdo poiché in tale caso la conica $K_{1}$ - che passa per i tre punti non allineati, $B_{1}, B_{2}, B_{3}$ - dovrebbe contenere come parte la $a$, la quale per ipotesi non passa per nessuno di detti punti.

Conviene in fine notare che le quartiche del tipo a) e $b$ ) rientrano come casi particolari nel tipo c) quando tutti e quattro i punti $B_{1}, B_{2}, B_{3}, B_{4}$, oppure solo due di essi, vengano a coincidere a coppie.

2) Il tipo generale di quartica $f_{4}$ degenere in una coppia di rette $r$ e $r^{\prime}$ e in una conica $K$ si ottiene assai facilmente.

Si osservi anzitutto che il punto $D$, comune alle due rette $r$ e $r^{\prime}$, deve risultare triplo se si vuole che le rette di un fascio $O$ seghino su questa quartica quaterne armoniche: pertanto la conica $K$ deve passare per detto punto.

Inoltre i due punti $B$ e $B^{\prime}$, ulteriori intersezioni della $K$ con le rette $r$ e $r^{\prime}$, devon esser punti doppi di cui un ramo abbia come tangente una retta per $O$ : si deduce che le rette $O B$ e $O B^{\prime}$ sono tangenti alla conica $K$.

Adunque la quartica più generale di questo tipo si costruisce nel modo seguente: si prenda una conica arbitraria $K$, e su essa un punto, pure arbitrario, $D$ : siano $r$ e $r^{\prime}$ due rette (arbitrarie) passanti per $D$ le quali segano la $K$ in due punti $B$ e $B^{\prime}$, indichiamo con $O$ il punto comune alle due tangenti in $B$ e $B^{\prime}$. La quartica costituita dalla conica $K$ e dalle rette $r$ e $r^{\prime}$ è la quartica richiesta: infatti su di essa le rette per $O$ segano quaterne tutte armoniche, essendovene 7 delle tali (quella segata dalla $O D$ che conta per 3 , e quelle segate dalle rette $O B$ e $O B^{\prime}$ ciascuna delle quali conta per 2).

3) Con facilità ancora maggiore si costruisce il tipo generale di quartica $f_{t}$, degenere in 4 rette, segata dalla rette per $O$ secondo quaterne armoniche. Basta infarti osservare che tale $f_{t}$ non puó possedere punti doppi (occorrerebbe che una delle tangenti principali, cioè una delle rette che compongono la $f_{+}$, passasse per $O$ ) e quindi le quattro rette passano tutte per un punto $F$, formando una quaterna armonica di raggi. Viceversa ogni quaterna armonica di raggi di un fascio $F$ costituisce una quartica $f_{4}$ segata dalle rette di qualsiasi fascio $O$ secondo quaterne armoniche; ed è inutile no- 
tare come questa quartica $f_{+}$appaia qualc caso particolare di quelle segate dalle rette di un fascio secondo quaterne il cui invariante abbia un valore costante affatto generico. Bisogna peró ricordare the una quartica siffatta non di origine ad un fascio di cubiche armoniche $(\$ 3)$.

\section{$\int 7$}

\section{Caso in cui il centro del fascio appartenga alla quartica.}

Per completare l'analisi delle quartiche segate dalle rette di un fascio secondo quaterne il cui invariante abbia un valore fisso, ci resta da esaminare il caso in cui il punto $O$, sostegno di detto fascio, appartenga alla quartica $f_{f}$. Come già ahbiamo osservato, se il punto $O$ fosse doppio, i gruppi segati dalle rette per $O$ avrebbero tutti $J=\mathrm{I}$, e se invece $O$ fosse triplo, $J$ risulterebbe indeterminato. Dovremo dunque supporre che $O$ sia semplice per la quartica $f_{+}$la quale, a sua volta, è supposta - come sempre - priva di parti multiple. Esamineremo prima il caso in cui l'invariante $J$ abbia un valore generico, poi il caso equianarmonico ed infine l'armonico.

I) Suppongasi dunque che la quartica $f_{t}$ sia segata dalle rette per un suo punto $O$ secondo quaterne il cui invariante $J$ abbia un valore fisso $K$ diverso da o e da $\infty$.

Dall'analisi instaurata nel $\mathrm{n}^{\circ} 3$ risulta che la tangente nel punto semplice $O$, la quale deve assorbire 6 delle 12 tangenti che da un punto generico vanno alla quartica, si stacca dalla quartica stessa. Le altre 6 tangenti dovendosi pure riunire in una, occorre che la cubica residua abbia un punto triplo. Si ha cosi che la $f_{f}{ }^{\prime}$ si compone di una retta passante per $O$ e di altre tre rette concorrenti in un unico punto: ed é d'altra parte chiaro come una quartica siffatta sia segata dalle rette per $O$ secondo quiterne di cui il birapporto, e quindi l'invariante $J$, ha un valore costante. Conviene anche osservare come questo tipo di quartiche non rientri come caso particolare in quelli esaminati nel $n^{\circ}{ }_{4}$ in cui si supponeva $O$ esterno alla $f_{4}$.

2) Abbiamo gid osservato che se si impone alla quartica

$$
f_{4}=\frac{\partial^{2} \varphi_{3}}{\partial x_{3}^{2}} \varphi_{3}-\frac{I}{2}\left(\frac{\partial \varphi_{3}}{\partial x_{3}}\right)^{2}=0,
$$

che è la quartica generale segata dalle rette per $O=$ (OoI) secondo quaterne equianarmoniche, di passare per il punto $O$, essa viene ad acquistare un punto quadruplo in $O$ : esistono invece quartiche segate dalle rette di un fascio - il cui sostegno sia un punto semplice della curva - le quali quindi non entrano come caso particolare in quelle di cui sopra abbiamo riferito l'equazione. Determiniamole.

A tale oggetto ricordiamo che se un gruppo di punti è equianarmonico ciascun suo punto è punto unito per le due proiettività cicliche del terz'ordine (una inversa dellaltra) che permutano gli altri tre: dunque nel nostro caso possiamo considerare sopra ogni retta $o$, passante per $O$, una proiettività ciclica del terz'ordine che lascia fisso $O$ e 
permuta le altre tre intersezioni di o con la $f_{4}$. Questa omografia dovra anche lasciar fissa la terna polare di $O$ rispetto la quaterna, terna che contiene il punto $O$ stesso contato una volta: pertanto tale terna si comporrà del punto $O$ e dell'altro punto unito della suddetta proiettiviti, contato questo due volte. Si deduce che la cubica polare di $O$ (rispetto la $f_{4}$ ) si compone di una retta a contata due volte e della tangente in $O$. Preso questo punto $O$ come punto (OOI), e assunta la tangente in $O$ come retta $x_{1}=0$, e la retta a come retta $x_{3}=0$, si deduce per la $f_{4}$ un'equazione del tipo

$$
f_{4}=x_{3}^{3} x_{1}+\varphi_{4}\left(x_{1} x_{2}\right)=0
$$

essendo $\psi_{4}$ una forma del quart'ordine nelle $x_{1} x_{2}$; ed è facile riconoscere che una quartica siffatta è segata dalle rette per $O=$ (OOI) secondo quaterne equianarmoniche.

La $f_{+}$ha in $O$ un flesso del second'ordine.

Si osservi ora che se $p_{4}$ si annulla per $x_{x}=0$, dalla quartica si stacca la retta $x_{\mathrm{r}}=0$ e resta una cubica equianarmonica; inoltre se le quattro radici di $\varphi_{4}=0$ sono distinte, la retta $a$ incontra la quartica in 4 flessi le cui tangenti passano per $O$; se due di esse coincidono si ha una cuspide $C$ di cui $C O$ è la tangente cuspidale; se ne coincidono tre si ha un punto triplo $T$, a tangenti distinte fra loro e dalla $O T$; infine se coincidono tutte e quattro si ha un punto triplo $T^{\prime}$, le cui tre tangenti principali coincidono nella $O T^{\prime}$ dando origine ad un ramo del terz'ordine. Osserviamo che la possibilita di queste singolarità si sarebbe potuta desumere a priori in base ai nostri teoremi generali indipendentemente dalla equazione sopra scritta.

3) Ci restano in fine da esaminare le quartiche $f_{4}$ segate dalle rette di un fascio, il cui sostegno $O$ sia un punto semplice per la curva, secondo quaterne armoniche.

Si consideri il punto $O^{\prime}$, coniugato armonico di $O$ rispetto gli altri due punti di una quaterna segata da una retta $o$ per $O$ : al variare di $o, O^{\prime}$ descriverà una retta oppure una conica per $O$ : si esclude questo secondo caso osservando che la quartica deve avere in $O$ un flesso, poichè la tangente in $O$ deve assorbire un numero di tangenti multiplo di 3 .

Resta cosi stabilito che la quartica $f_{4}$ si compone di una retta a (luogo dei punti $O^{\prime}$ ) e di una cubica residua $C_{3}$ avente un flesso in $O$. Essendo armonici i gruppi segati dalle rette per $O$, si deduce che la retta $a$ è la polare armonica del flesso $O$.

Viceversa ogni quartica composta di una cubica $C_{3}$, e della polare armonica di uu suo flesso $O$, è segata effettivamente dalle rette per $O$ secondo quaterne tutte armoniche.

Si osservi che la cubica $C_{3}$ puó comunque degenerare, senza peró acquistare un punto doppio in $O$ : in particolare da essa puó staccarsi una retta per $O$, e anche la conica residua puó ridursi ad una coppia di rette, col che la quartica $f_{4}$ si riduce ad una retta per $O$ e ad una terna di rette passanti per un medesimo punto $C$, questa terna e la $C O$ formando un gruppo armonico.

Giova in fine notare come il tipo di quartiche ora esaminato non rientri quale caso particolare in nessuno di quelli studiati nel $n^{\circ} 6$, in cui supponevasi il punto $O$ esterno alla quartica. 


\section{$\$ 8$.}

\section{Determinazione dei fasci di cubiche a modulo costante.}

Ora che abbiamo determinato e costruito tutti i tipi di quartiche segate dalle rette di un fascio secondo quaterne a birapporto costante, converrà ritornare al problema da cui avevamo preso le mosse, cioè al problema di classificare i fasci di cubiche di ugual modulo i quali corrispondono appunto ai fasci di raggi suddetti.

A tal uopo conviene premettere le seguenti tre osservationi.

I) $\dot{E}$ noto che, dato un fascio di cubiche (e cosi di curve di un ordine qualsiasi)

$$
\lambda \varphi\left(x_{1} x_{2} x_{3}\right)+\mu \cdot \psi\left(x_{1} x_{2} x_{3}\right)=0,
$$

i punti doppi delle curve del fascio si ottengono annullando contemporaneamente $\mathrm{i}$ minori estratti dalla matrice

cioè intersecando le due curve

$$
\left|\begin{array}{lll}
\frac{\partial \psi}{\partial x_{1}} & \frac{\partial \varphi}{\partial x_{2}} & \frac{\partial p}{\partial x_{3}} \\
\frac{\partial \psi}{\partial x_{1}} & \frac{\partial \psi}{\partial x_{2}} & \frac{\partial \psi}{\partial x_{3}}
\end{array}\right|,
$$

$$
\begin{aligned}
& \frac{\partial \varphi}{\partial x_{2}} \frac{\partial \psi}{\partial x_{3}}-\frac{\partial \varphi}{\partial x_{3}} \frac{\partial \psi}{\partial x_{2}}=0 \\
& \frac{\partial \varphi}{\partial x_{3}} \frac{\partial \psi}{\partial x_{1}}-\frac{\partial \varphi}{\partial x_{1}} \frac{\partial \psi}{\partial x_{3}}=0
\end{aligned}
$$

e togliendo i punti comuni alle due curve

$$
\frac{\partial \varphi}{\partial x_{3}}=0, \quad \frac{\partial \psi}{\partial x_{3}}=0
$$

Si riconosce in tal modo che in un fascio di cubiche esistono in generale $12 \mathrm{cu}-$ biche dotate di punto doppio: questo numero puo peró diminuire quando qualcuno dei punti doppi cada in un punto base del fascio, o sia un punto di singolarità più elevata (cuspide, tacnodo, ...), in modo che la cubica cui tale punto doppio appartiene assorba un certo numero $r>\mathrm{I}$ di cubiche dotate di punto doppio. Precisamente facili sviluppi analitici, $i$ quali costituiscono una verifica diretta, inutile qui a ripetersi, permettono di riconoscere che:

a) Una cubica, $C$, dotata di cuspide fuori dei punti base, conta come 2 cubiche dotate di punto doppio;

b) ove la cuspide di $C$ cada in un punto base, purchè la tangente cuspidale resti distinta dalla tangente fissa che ivi hanno le curve del fascio, la $C$ conta come $3 \mathrm{cu}$ biche dotate di punto doppio;

c) se invece la tangente cuspidale riesce tangente fissa, la $C$ conta 4 volte.

d) Una cubica, $C$, dotata di tacnodo, fuori dei punti base, assorbe 3 cubiche dotate di punto doppio; 
e) se il tacnodo di $C$ cade in un punto base, restando la tangente tacnodale diversa dalla tangente fissa che ivi hanno le cubiche del fascio, la $C$ conta 4 volte nel numero delle cubiche con nodo;

f) se per di più la tangente tacnodale coincide con la tangente fissa, la $C$ conta 6 volte.

g) Una cubica, $C$, dotata di un punto triplo fuori dei punti base, conta 4 volte nel gruppo delle cubiche nodate;

b) se il punto triplo cade in un punto base, la $C$ conta 6 volte; conta di più ove una delle tre rette che la formano risulti tangente fissa.

i) Una cubica, $C$, la quale contenga come parte una retta contata due volte, assorbe 6 cubiche dotate di nodo.

2) Sia data una quartica $f_{4}$, quartica di diramazione del piano doppio definito da una rete di cubiche $C$ passanti per 7 punti base: una retta $r$ sega la quartica $f_{4}$ in quattro punti che corrispondono ai quattro punti doppi della $g_{2}^{1}$ che le cubiche della rete suddetta scgano sulla cubica $C_{r}$ corrispondente alla retta $r$. Pertanto alle rette di un fascio, il cui sostegno $O$ appartenga semplicemente alla quartica $f_{+}$, corrispondono le cubiche di un fascio le quali hanno fra loro un contatto nel punto $O^{\prime}$ in cui coincidono i due omologhi di $O$. In particolare alla retta tangente alla quartica nel punto $O$ (supposta questa tangente a contatto bipunto) corrisponde una cubica avente in $O^{\prime}$ un punto doppio. Ciò si dimostra osservando che, sopra una cubica $C$, ogni $g_{2}^{\mathrm{I}}$ è segata dalle rette di un fascio il cui sostegno $U$ appartiene alla cubica stessa: se due coincidenze della $g_{2}^{1}$ vengono infinitamente vicine esse cadono in un punto doppio della curva il quale assorbe precisamente due delle tangenti uscenti da $U$.

Se invece la tangente $r$ viene ad avere nel punto di contatto $O$ tre intersezioni con la quartica, ad essa corrisponde una cubica avente una cuspide nel punto $O^{\prime}$ omologo di $O$, cuspide che assorbe precisamente tre coincidenze della $g_{2}^{t}$ suddetta. In fine se la $r$ ha un contatto quadripunto con la quartica, la cubica ad essa corrispondente viene ad avere in $O^{\prime}$ un tacnodo il quale assorbe tutte le quattro coincidenze della $g_{2}^{\mathrm{r}}$; si esclude infatti che la $r$ possa rappresentare una cubica con punto triplo poiche in tal caso [osservazione precedente, capover'so $g$ )] essa conterebbe quattro volte fra le tangenti alla quartica $f_{4}$ condotte da un punto generico della $r$ stessa.

Sia ora $O$ un punto doppio della quartica e sia a tangenti distinte: le rette per $O$ corrispondono a cubiche, di un fascio, dotate di un punto doppio $O^{\prime}$, e le tangenti principali (avendo tre intersezioni con la quartica) corrispondono alle due cubiche cuspidate appartenenti al fascio suddetto. Si osservi che il punto $O^{\prime}$, in cui cadono quattro intersezioni delle cubiche del fascio, deve essere uno dei sette punti base della rete che definisce il piano doppio.

Se invece il punto $O$ diviene una cuspide di $f_{4}$, allora la cubica $C_{r}$, omologa della tangente cuspidale $r$, possiede ancora una cuspide in un punto base della rete: poiché la $r$ assorbe quattro delle tangenti alla $f_{4}$ uscenti da un suo punto $P$, la $C_{r}$ dovrà contare quattro volte nel numero delle cubiche dotate di nodo appartenenti al 
fascio omologo di $P$, e pertanto la tangente cuspiciale di $C_{r}$ dovia essere una tangente fissa per detto fascio. (Cfr. Osservazione $\mathrm{I}^{\mathrm{a}}$ ).

Vediamo ora cosa corrisponda ad una retta $r$ che sia tangente tacnodale alla quartica $f_{4}$ in un punto $O$. Siccome la $r$ assorbe sei delle tangenti alla $f_{+}$uscenti da un suo punto $P$, cosi la cubica $C_{r}$, omologa di $r$, potrà essere:

o una cubica dotata di tacnodo, la cui tangente tacnodale sia fissa per le cubiche del fascio corrispondente ad un punto, $P$, di $r$;

o una cubica dotata di punto triplo (a tangenti distinte) posto in un punto base del fascio predetto;

$o$, in fine, una cubica dotata di una retta doppia.

Ciò si deduce in base ai risultati riferiti nell'Osservazione 1 ); vedremo poi come questi tre tipi differenti di $C_{r}$ diano effettivamente origine ad una retta $r$, tangente tacnodale di $f_{4}$.

Nota. - Il risultato precedente puó a prima vista meravigliare, dovendosi supporre a priori che ad un unico tipo di retta $r$ corrisponda un unico tipo di cubica $C_{r}$. Ma la cosa riesce chiarita da considerazioni di limite cui brevemente accenniamo.

Si consideri dapprima il tacnodo $O$ come limite di due punti doppi della quartica, $\mathrm{O}_{\mathrm{r}}$ e $\mathrm{O}_{2}$; la tangente tacnodale $r$ apparirà come la congiungente due punti doppi infinitamente vicini.

Alla retta $r$ corrisponderd una cubica dotata di due punti doppi $O_{1}^{\prime}$ e $O_{2}^{\prime}$ : quando $O_{1}$ e $O_{2}$ diventano infinitamente vicini, anche $O_{1}^{\prime}$ e $O_{2}^{\prime}$ diventeranno infinitamente vicini, oppure andranno su una curva fondamentale per la trasformazione $[\mathrm{I}, 2]$ che definisce il piano doppio. Nel primo caso la $C_{r}$ viene ad avere un tacnodo la cui tangente tacnodale $O_{1}^{\prime} O_{2}^{\prime}$, contenendo due punti base della rete, è tangente fissa per le cubiche della rete; nel secondo caso la $C_{r}$ viene a contenere la $O_{1}^{\prime} O_{2}^{\prime}$ come retta doppia.

Si consideri invece il tacnodo $O$ come limite di una cuspide: alla retta $r$ (supposta ancora tangente cuspidale) corrisponde una cubica $C_{r}$ dotata di cuspide, e tale che la tangente cuspidale è tangente fissa per il fascio di cubiche corrispondente ad un punto $P$ di $r$.

Ciò posto consideriamo una cubica $C$ variabile al variare di certi parametri $a, b, \ldots, e$ :

$$
a y^{2}+b x^{3}+c x^{2} y+d x y^{2}+e y^{3}=0
$$

la quale abbia una cuspide nel punto $O=(00)$ con tangente cuspidale fissa $y=0$. Se la quarta coincidenza della $g_{2}^{\prime}$ segata sulla $C$ dalle parallele all'asse $y$ viene a cadere nella cuspide, allora si ha $a=0$, oppure $b=0$; nel primo caso la cubica si riduce a tre rette passanti per $O$ e comunque disposte, nel secondo caso invece si spezza nella retta $y=0$ e in una conica ivi tangente alla retta stessa.

Pertanto quando la cuspide di $f_{+}$diviene un tacnodo, la cubica $C_{r}$, omologa alla tangente cuspidale $r$, si ridurra ad una terna di rette passanti per un punto base della rete, ma comunque disposte, oppure ad una cubica degenere in una retta, tangente fissa per la rete, ed in una conica a questa stessa retta tangente. 
3) Abbiamo già osservato che $i$ fasci di rette il cui sostegno $O$ giaccia sulla quartica $f_{4}$ rappresentano fasci di cubiche (appartenenti alla nostra rete di grado 2 che definisce il piano doppio) i cui ulteriori due punti base riescono infinitamente vicini. Da ciò segue che, se il fascio di cubiche a modulo costante non ha tutti i nove punti base infinitamente vicini, si puó sempre determinare la rete di cubiche definitrice del piano doppio in modo che il detto fascio corrisponda ad un fascio di rette il cui sostegno $O$ risulti esterno alla quartica di diramazione.

Converrà quindi esaminare anzitutto quando un fascio di cubiche, $\mathrm{i}$ cui nove punti base siano tutti infinitamente vicini, risulti costituito di cubiche a modulo costante (diverso da I).

Siano adunque $f$ e $\varphi$ due cubiche aventi in un punto $P$ un contatto 9-punto: sia

$$
\psi=f+\lambda_{0} \rho
$$

la cubica del fascio che ha in $P$ un punto doppio che sard una cuspide, o singolarità superiore, avendo $\psi$ un modulo diverso da I. Siccome $\psi$ ed $f$ devono avere 9 intersezioni riunite in $P$, ma non una parte comune, si deduce che la $\psi$ ha in $P$ un punto triplo, e precisamente si compone di tre rette coincidenti in una che risulta tangente di flesso per la $f$.

Assumendo $P$ come punto all'infinito dell'asse $y$, la polare armonica di $P$ (rispetto ad $f$ ) come asse $x$, e la tangente in $P$ come retta all'infinito, le cubiche del fascio risultano definite dall'equazione

$$
y^{2}+f_{3}(x)+\lambda=0
$$

dove $f_{j}(x)$ è un polinomio di terzo grado in $x$, e $\lambda$ un parametro.

Per tal modo si vede che le cubiche del fascio hanno lo stesso birapporto che le quaterne di punti costituite dal punto all'infinito dell'asse $x$ e della terna variabile $f_{3}(x)+\lambda=0$.

Queste quaterne formano una $g_{+}^{\mathrm{i}} \mathrm{i}$ cui gruppi hanno per ipotesi un modulo costante, quindi vi è un gruppo che possiede un punto triplo (distinto da $x=\infty$ ): assunto questo punto come punto $x=0$, la detta $g_{+}^{1}$ ha l'equazione

$$
x^{3}+\mu=0 \text {, }
$$

ed è quindi costituita di tutti gruppi equianarmonici.

Concludendo: l'unico fascio di cubiche a modulo costante $i$ cui nove punti base siano infinitamente vicini è il fascio

$$
y^{2}+x^{3}+\lambda=0
$$

costituito tutto di cubiche equianarmoniche: esso si ottiene combinando linearmente una cubica equianarmonica e una sua tangente di flesso contata tre volte.

Ciò posto veniamo alla determinazione dei fasci di cubiche a modulo costante, e cominciamo dai

I. Fasci di cubiche il cui modulo costante abbia un valore generico. - Per quanto abbiamo visto (Osservazione 3 ) questo fascio puó essere supposto rappresentato da un 
fascio di rette il cui sostegno $O$ sia esterno alla quartica $f_{4}$. Adunque $\left(n^{\circ} 4\right)$ ad ogni fascio di cubiche a modulo costante (di valote generico) corrisponde, come quartica $f_{4}$, una quartica dotata di due tacnodi $T_{1}$ e $T_{2}$, cioè costituita da una coppia di coniche $K_{1}$ e $K_{2}$ fra loro bitangenti; le cubiche del fascio riescono rappresentate dalle rette passanti per il punto $O$, intersezione delle tangenti comuni a $K_{1}$ e $K_{2}$ nei punti $T_{\text {I }}$ e $T_{2}$. Ma esistono due famiglie di fasci di cubiche corrispondenti a quest'unico tipo di quartica $f_{4}$; abbiamo infatti notato (Osservazione 2) che alla tangente tacnodale di una quartica di diramazione puó corrispondere:

$I^{0}$ o una cubica dotata di tacnodo, la cui tangente tacnodale sia tangente fissa per tutte le curve del fascio;

$2^{\circ}$ o una cubica dotata di un punto triplo, posto in un punto base del fascio, le cui tre tangenti principali sian distinte fra loro e dalla tangente fissa (che ivi hanno le curve del fascio);

$3^{\circ}$ o una cubica contenentc come parte una retta contata due volte.

Ora si riconosce facilmente che se il nostro fascio deve contenere due cubiche di uno dei tre tipi precedenti, queste saranno o entrambe del tipo $1^{\circ}$, oppure una del tipo $2^{\circ}$ e l'altra del tipo $3^{\circ}$ : infatti, nelle altre ipotesi, il fascio sarcbbe costituito di cubiche aventi tutte un punto doppio o - in particolare - una parte in comune.

Si conclude pertanto che:

esistono due famiglie di fasci di cubiche il cui modulo ba un valore generico costante; un fascio della prima famiglia è caratterizzato dal possedere due cubiche tacnodate le cui tangenti tacnodali sono tangenti fisse per il fascio; invece un fascio dell'altra famiglia è caratterizato dal possedere una cubica dotata di punto triplo in un punto base del fascio e una seconda cubica contenente una relta doppia (non passante per detto punto triplo).

Un fascio della prima famiglia si costruisce prendendo due coniche bitangenti $K_{1}$ e $K_{2}$ e le loro tangenti comuni $t_{1}$ e $t_{2}$ : il fascio è

$$
\lambda\left(K_{1}+t_{1}\right)+u\left(K_{2}+t_{2}\right)
$$

un fascio della seconda famiglia si costruisce combinando linearmente una cubica $2 a+b$, degenere in una retta $a$ contata due volte e in una retta $b$, con una seconda cubica $c+d+e$, riducibile in tre rette $c, d, e$, passanti per un medesimo punto di $b$ : il fascio è

$$
\lambda(2 a+b)+\mu(c+d+e) .
$$

Resta per tal modo confermato the le due famiglie suddette contengono ciascuna $\infty^{8}$ fasci, cosi come sono $\infty^{8}$ le quartiche $f_{+}$cui tali fasci corrispondono. Una facile verifica analitica mostra che effettivamente queste due diverse famiglie di fasci di cubiche corrispondono ad un unico tipo di quartica $f_{4}$, cioc ad una quartica, dotata di due tacnodi, costituita da due coniche bitangenti. Per riconoscere ció occorre prendere come rete che definisce il piano doppio:

nel primo caso, la rete delle cubiche passanti per il punto $\left(t_{1} t_{2}\right)$, aventi un contatto tripunto con $K_{\mathrm{I}}$ nel punto di tangenza $\left(K_{1} t_{2}\right)$ e parimente un contatto tripunto con $K_{2}$ nel punto di tangenza $\left(K_{2} t_{1}\right)$; 
nel secondo caso, la rete di cubiche passanti per il punto $(a e)$, tangenti alle $c, d$, nei punti $(a c),(a d)$ e tangenti alli $b$ nel punto $(b c)=(b d)=(b e)$.

II. Fasci di cubiche equiararmoniche. - Abbiamo visto che non esiste un tipo generale di quartica segata dalle rette di un fascio secondo quaterne equianarmoniche: tuttavia esiste un tipo generale di fasci di cubiche equianarmoniche, tipo che comprende come casi particolari tutti gli altri, anche il fascio di cubiche equianarmoniche i cui nove punti base siano infinitamente vicini.

Per dimostrare ciò si consideri la quartica

$$
f_{4}=\frac{\partial^{2} \varphi_{3}}{\partial x_{3}^{2}} \varphi_{3}-\frac{\mathrm{I}}{2}\left(\frac{\partial \varphi_{3}}{\partial x_{3}}\right)^{2}=0
$$

che è segata dalle rette per $O=(\mathrm{OOI})$ secondo quaterne tutte equianarmoniche (Cfr. $\mathrm{n}^{\circ} \mathrm{s}$ ) e la si faccia variare in guisa da acquistare un punto doppio, $P$, il che accade, per esempio, ove la cubica $P_{;}=0$ acquisti essa stessa un punto doppio $P$, risultando allora $P$ una cuspide per $f_{+}$di cui $O P$ è la tangente cuspidale. Le rette del fascio $O$ rappresentano ancora cubiche tutte equianarmoniche, e la retta $O P$ rappresenta una cubica dotata di punto doppio in un punto $B$, base della rete che definisce il piano doppio (precisamente $B$ è una cuspide la cui tangente cuspidale è fissa per le curve del fascio). Pertanto il fascio $\left|C_{0}\right|$ delle cubiche che corrispondono alle rette per $O$, ha due, fra $i$ nove, punti base infinitamente vicini $\mathrm{i}$ quali cadono nel punto $B$. Ora si prendano gli altri 7 punti base per definire una rete di grado 2 : otterremo un nuovo piano doppio, la cui quartica di diramazione, $f_{4}^{\prime}$, sari segata dalle rette corrispondenti alle cubiche di $\left|C_{\mathrm{o}}\right|$ secondo quaterne equianarmoniche, e queste rette passeranno tutte per il punto omologo di $B$, punto che cade sulla quartica $f_{+}^{\prime}$.

Vediamo cosi che, mentre la quartica $f_{4}^{\prime}$ non rientra come caso particolare nelle quartiche

$$
\frac{\partial^{2} \varphi_{3}}{\partial x_{3}^{2} \varphi_{3}}-\frac{I}{2}\left(\frac{\partial \varphi_{3}}{\partial x_{3}}\right)^{2}=0,
$$

il fascio di cubiche equianarmoniche a cui essa corrisponde appare come caso particolare di quello relativo ad una

$$
f_{1}=\frac{\partial^{2} \psi_{3}}{\partial x_{3}^{2} \varphi_{3}}-\frac{1}{2}\left(\frac{\partial r_{3}}{\partial x_{3}}\right)^{2}=0 .
$$

Sicchè tutti $\mathrm{i}$ fasci di cubiche cquianarmoniche $\mathrm{i}$ quali diano origine a quartiche che rientrano, come casi particolari, nel tipo dclla $f_{+}^{\prime}$, appaiono essi stessi come casi particolari del fascio di cubiche corrispondenti alla quartica

$$
f_{4}=\frac{\partial^{2} \psi_{3}}{\partial x_{3}^{2}} p_{3}-\frac{I}{2}\left(\frac{\partial p_{3}}{\partial x_{3}}\right)^{2}=0
$$

dove $\varphi_{3}=0$ è una cubica affatto generale. Oa tale quartica è dotata di 6 flessi le cui tangenti passano per il punto $O=$ (OOI) che rappresenta il fascio di cubiche equianarmoniche; le tangenti di flesso corrispondono a cubiche dotate di una cuspide fuori dei punti base. Pertanto (Cfr. $n^{i} 5,6$ ) possiamo concludere affermando che: 
Il fascio piu generale di cubiche equianarmoniche è caratterizzato dal possedere $6 \mathrm{cll}$ biche dotate di cuspide (fuori dei punti base); ogni altro fascio appare come limite di questo quando due, o più, cubiche cuspidate risultano fra loro infinitamente vicinc. Questi fasci sono $\infty^{11}$ come le quartiche $f_{4}$ cui corrispondono.

La costruzione diretta dei fasci di cubiche equianarmoniche risulterebbe molto complicata, ma quanto abbiamo detto ne di una costruzione indiretta attraverso la quartica $f_{4}$ e il relativo piano doppio; la considerazione di questa quartica, tenuto conto dei risultati dell'Osservazione I) di questo paragrafo, permette anche di riconosecre facilmente i caratteri dei vari fasci particolari. Qui ci limiteremo a notare che, come risulta dall'esame della $f_{4}$, acciocchè il fascio sia costituito da cubiche tutte equianarmoniche basta che esso possegga 5 cubiche cuspidate; l'esistenza di una sesta cubica cuspidata ne viene come conseguenza.

Nota I. - Ricordiano che, data una cubica equianarmonica, esistono delle omologie e omografie cicliche del terz'ordine, quest'ultime coi punti uniti sopra la cubica, le quali la lasciano invariata: anzi ciascuna di questa due proprieti caratterizza le cubiche equianarmoniche.

Ora si puó chiedere se, dato un fascio di cubiche equianarmoniche, esiste qualche omologia od omografia della specie suddetta, la quale lasi invariate tutte le cubiche del fascio. Alla quale domanda la risposta è negativa: i fasci di cubiche le quali siano invarianti per una medesima omologia ciclica del terz'ordine o per una omografia pure ciclica del terz'ordine (i cui punti uniti giacciono sulla curva stessa) sono fasci di cllbiche equianarmoniche particolari. E la loro costruzione si ottiene immediatamente come segue.

Le cubiche del fascio $\lambda f+\mu p$ siano invarianti per un'omologia $\omega$, ciclica del terz'ordine, di centro $O$ e asse $a$. Allora, come è ben noto, le intersezioni della $a$ con una cubica del fascio sono flessi per questa e le tangenti di flesso passano per $O$. Il fascio è dunque ottenuto combinando linearmente la retta $a$ contata tre volte e una terna di rette per $O$. Pertanto, relativamente ad una medesima omologia $\omega$, esistono $\infty^{3}$ fasci di cubiche invarianti costituiti dalle cubiche del tipo

$$
\lambda_{0} y^{3}+\left(\lambda_{1} x^{3}+\lambda_{2} x^{2}+\lambda_{3} x+\lambda_{4}\right)=0
$$

sicchè i fasci di tal natura appaiono essere in tutto $\infty^{7}$.

Suppongasi, invece, che ciascuna cubica del fascio $\lambda f+\mu \%$ sia invariante per un'omografia $\omega$, ciclica del terz'ordine, i cui tre punti uniti $A, B, C$, giacciono sulla cubica stessa. Questi tre punti uniti saranno punti base del fascio, e ivi la tangente di ciascuna cubica, dovendo rastar fissa per la w, coinciderà con una delle rette unite che vi passano, e sari tangente comune per tutte le curve del fascio: resteranno cosi, fuori dei punti $A, B, C$, altri tre punti base del fascio, i quali costituiranno un ciclo della omografia w. Si riconosce poi facilmente che presi i tre punti $P, Q, R$ di un ciclo arbitrario della w, le cubiche passanti per essi, per $A, B, C$, c tangenti in questi ultimi alle rette $A B, B C, C A$ formano effettivamente un fascio, cui appartengono tre cubiche spezzate in una retta $\mathrm{e}$ in una conica: una di queste consta della retta $C$ d e della conicr pas- 
sante per $A, B, P, Q, R$, l'altra della retta $A B$ e della conica $B C P Q R$, e l'ultima. della retta $B C$ e della conica $C A P Q R$.

Queste tre cubiche risultando invarianti per la $\omega$, tutte le cubiche del fascio restano fissc per la (1) stessa, e sono quindi equianarmoniche. Tale fascio fu incontrato da S. Kantor esaminando le proiettiviti che lasciano fermo un fascio di cubiche ${ }^{4}$ ); erroneamente poi egli aggiunge che sono anche composti di cubiche equianarmoniche $\mathrm{i}$ fasci i cui nove punti base costituiscano tre cicli di una proiettivita ciclica del terz'ordine, fasci nei quali il precedente figura come caso particolare ${ }^{5}$ ).

Aggiungasi che, ove il triangolo $A B C$ sia assunto come triangolo fondamentale delle coordinate, l'equazione di una qualsiasi cubica invariante per la $\omega$ è della forma

$$
\lambda_{0} x_{2} x_{3}^{2}+\lambda_{1} x_{3} x_{1}^{2}+\lambda_{2} x_{1} x_{2}^{2}=0
$$

sicchè appare che le suddette cubiche equianarmoniche formano una rete, e ciascuno degli $\infty^{2}$ fasci di questa risulta determinato dalla condizione di avere come punti base i tre punti $P, Q, R$ di un ciclo (arbitrario) della $\omega$.

Pertanto i fasci di cubiche equianarmoniche che restino invariate per una proiettiviti ('), cicica del terz'ordine, risultano $\infty^{\circ}$, essendo $\infty^{6}$ il numero delle suddette proiettivita.

Si conclude che $\mathrm{i}$ fasci di cubiche invarianti per omologie od omografie del terz'ordine rientrano come casi particolari entro la faniglia degli $\infty^{\prime \prime}$ fasci di cubiche equianarmoniche che abbiamo determinato.

III. Fasci di cubiche armoniche. - Per quanto abbiamo notato all'Osservazione 3) i fasci di cubiche armoniche posson essere rappresentati sempre da fasci di rette il cui sostegno è esterno alla quartica di diramazione $f_{4}$. Adunque l'analisi fatta nel $\mathrm{n}^{\mathrm{o}} 6$ delle quartiche segate dalle rette di un fascio secondo quaterne equianarmoniche, tenuto conto dell'osservazione 2) di questo paragrafo, ci permette di concludere:

Esistono tre famiglie di fasci di cubiche tutte armoniche le quali sono cosi caratterizzate :

i fasci della prima famiglia contengono quattro cubiche dotate di tacnodo (fuori dei punti base);

i fasci della seconda famiglia contengono due cubiche dotate di tacnodo (fuori dei punti base) e due cubiche dotate di cuspide nei punti base (per modo che la tangente cuspicale non sia tangente fissa per le cubiche del fascio);

i fasci della terza famiglia contengono quattro cubiche con cuspide (la cui tangente cuspidale non è tangente fissa per le curve del fascio).

4) 1. c. ${ }^{1}$ ), pp. 6, 9 .

5) Per mettere in luce l'errore di Kantor basta costruire un fascio di cubiche passanti per i tre cicli di una proiettività ciclica del terz'ordine e non costituito da cubiche equianarmoniche: tale è il fascio definito da una cubica con nodo, e da una terna di rette costituenti un ciclo della proiettività del terz'ordine che lascia ferma la cubica suddetta. 
I fasci di ciascuna famiglia sono $\infty^{9}$, come le quartiche cui corrispondono; ogni fascio di cubiche armoniche rientra come caso particolare in una delle famiglie precedenti ove risultino infinitamente vicine aliune delle quattro cubiche singolari nominate. La costruzione diretta di tali fasci richiederebbe laboriosi sviluppi di calcolo, ma le considerazioni svolte ce ne dànno, come per $\mathrm{i}$ fasci di cubiche equianarmoniche, una costruzioni indiretta attraverso la quartica $f_{4}$ e il relativo piano doppio.

Nota II. - Ricordiamo che, data una cubica armonica, esistono delle omografie cicliche del quart'ordine le quali la lasciano invariata: una tale omografia ha come punto unito un flesso $F$ della cubica e come rette unite la tangente di flesso, la polare armonica di $F$, e una delle altre tre tangenti uscenti da $F$ stesso, il cui punto di contatto risulta essere un secondo punto unito.

E noto inoltre come la suddetta proprietà caratterizzi le cubiche armoniche. Ora, analogamente a quanto accade per le cubiche equianarmoniche, si domanda se esiste qualche omografia ciclica del quart'ordine la quale lasci ferma ciascuna cubica di un fascio generico di cubiche armoniche. Anche qui la risposta è negativa: i fasci di cubiche armoniche, le quali siano invarianti per una medesima omografia ciclica del quartordine sono fasci particolari. E la loro costruzione si ottiene come segue.

Sia $\lambda f+\mu p$ un fascio di cubiche tutte trasformate in sé da una proicttivita $\omega$, ciclica del quart'ordine.

Per quanto sopra abbiamo ricordato, se $F, G, H$ sono i tre punti uniti della (w), uno di essi per es. $F$, sard un flesso comune a tutte le cubiche, e la retta opposta, $G H$, sarà la relativa polare armonica.

Parimenti una delle rette $F G$ e $F H$, per es. la $F H$, sarà tangente di flesso per tutte le cubiche, le quali risulteranno pure tangenti in $G$ alla retta $F G$. Preso $F$ come punto all'infinito dell'asse $y, G$ come punto (oo) e $G H$ come asse $x$, le cubiche del fascio, essendo per ipotesi armoniche, avranno un'equazione del tipo

$$
\lambda_{0} y^{2}+\lambda_{1} x^{3}+\lambda_{2} x=0 .
$$

Pertanto esiste una rete di cubiche armoniche invarianti per l'omografia $\omega$, ciclica del quart'ordine; questa rete contiene $\infty^{2}$ fasci, ciascuno dei quali è determinato dai quattro punti base, fuori di $F$ e $G$, i quali costituiscono un ciclo della $\omega$.

Dipendendo la $\omega$ da sei parametri arbitrari (le coordinate dei punti $F, G, H$ ) i fasci di cubiche armoniche le quali restano invariate per un'omografia ciclica del quart'ordine sono $\infty^{8} 6$ ).

Si riconosce facilmente che uno di questi fasci contiene tre cubiche singolari: una

6) Di questo tipo sembra fossero i fasci di cubiche armoniche, che il Caporali [Rendiconti della R. Accademia delle Scienze fisiche e matematiche di Napoli, anno XXII (1883), p. 3I4] nella sua relazione sulla citata memoria di KanTor dice esser stati scoperti dal KanTor stesso. Ma di ció non ho trovato traccia nell'esame dei fasci di cubiche invarianti per proiettività cicliche che Kavtor svolge nella sua memoria, pubblicata cinque anni più tardi. 
degenere in tre rette per il punto base $F$, una seconda dotata di una cuspide in $G$, con tangente cuspidale $G H$, una terza composta della retta $F H$ ed una conica ad essa tangente in $H$. Questo fascio appare dunque come un fascio particolare della seconda famiglia ove una cubica dotata di cuspide e una di tacnodo divengano infinitamente vicine, dando origine alla cubica con punto triplo in $F$.

Bologna, gennaio 1916.

OSCar ChISINI. 